\title{
Coulomb Effect: A Possible Probe for the Evolution of Hadronic Matter
}

\author{
T. Osada* and Y. Hama ${ }^{\dagger}$ \\ Instituto de Física, Universidade de São Paulo, CP 66318, \\ 05315-970 São Paulo -SP, Brazil
}

(November 13, 2018)

\begin{abstract}
Electromagnetic field produced in high-energy heavy-ion collisions contains much useful information, because the field can be directly related to the motion of the matter in the whole stage of the reaction. One can divide the total electromagnetic field into three parts, i.e., the contributions from the incident nuclei, non-participating nucleons and charged fluid, the latter consisting of strongly interacting hadrons or quarks. Parametrizing the space-time evolution of the charged fluid based on hydrodynamic model, we study the development of the electromagnetic field which accompanies the high-energy heavy-ion collisions. We found that the incident nuclei bring a rather strong electromagnetic field to the interaction region of hadrons or quarks over a few fm after the collision. On the other hand, the observed charged hadrons' spectra are mostly affected (Coulomb effect) by the field of the charged fluid. We compare the result of our model with experimental data and found that the model reproduces them well. The pion yield ratio $\pi^{-} / \pi^{+}$at a RHIC energy, $\mathrm{Au}+\mathrm{Au} 100+100 \mathrm{GeV} /$ nucleon, is also predicted.
\end{abstract}

PACS: $25.75 .-\mathrm{q}$

\section{INTRODUCTION}

Most of the important informations on the new phase of quark-gluon matter are obtained by investigating their space-time development in high-energy heavy-ion collisions. These studies promise not only to specify the thermodynamical conditions for the opening new phase but also to get useful knowledge of the evolution of the system. The Bose-Einstein correlations [1] attract a great deal of attention as a powerful tool to study the geometry of the particle emitting sources. However, very complicated interactions characteristic of these collisions prevent us from drawing a clear image of the sources.

The Coulomb interaction between a frozen out hadron and the strongly interacting charged hadronic matter may also be helpful in studying the evolution of the hadronic matter [2 9]. One of the advantages of studying its effects is that there is no ambiguity in constructing the electromagnetic fields if one knows completely the motion of the hadronic or quark matter. Conversely, if one understands the electromagnetic fields precisely, one can know the behavior of the hadronic or quark matter in the high-energy heavy-ion collisions. Another advantage is that the electromagnetic fields carry information over the whole duration of the nuclear collision (actually, even of the stage before the collision.) Fortunately, a great number of charged hadrons are produced in the high-energy heavy-ion collisions. It is expected that one can obtain information on the electromagnetic fields by carefully studying their momentum distributions. The importance of the Coulomb effect at Bevalac energies has been pointed out by several authors [2].

The hydrodynamic model [10,11] describes the space-time development of the hadronic matter and it can explain various experimental results. The purpose of this paper is to explore this model a little further into the problem of the electromagnetic fields due to the expanding charge distribution produced in high-energy heavy-ion collisions.

In the following, we will confine ourselves only to symmetrical collisions such as $\mathrm{Pb}+\mathrm{Pb}$. The extension to nonsymmetrical cases is straightforward. We will begin by describing in the next section how to compute the number of participant nucleons and their distribution in the transverse plane. In order to study the space-time development of the produced electromagnetic field, we shall assume a longitudinal scaling solution without transverse expansion for the charged fluid. We find this will be enough to reproduce the main features of the electromagnetic field in question and moreover, in computing the ratios of the yields (for instance $\pi^{-} / \pi^{+}$), possible deviations are largely canceled out. In Section III, we give account of how to estimate the net charge distribution inside the fluid along the collision axis. This will be done by using various experimental results on $\mathrm{Pb}+\mathrm{Pb} 158 \mathrm{GeV} /$ nucleon in the CERN-SPS. Then, in

*e-mail: osada@fma.if.usp.br

${ }^{\dagger}$ e-mail: hama@fma.if.usp.br 
Section $\mathrm{I}$, the electromagnetic field produced by this expanding fluid is computed. In addition to this, fields by both the incident nuclei and by the penetrating systems consisting of spectator nucleons are also taken into account $[$. The determination of the freeze-out hypersurface and computation of the transverse-momentum distribution is given in Section V. The results obtained are compared with the experimental data for the pionic yield ratio $\pi^{-} / \pi^{+}$by NA44 Collaboration [12]. Finally, we close our discussions with some concluding remarks and prediction for the pion yield ratio for $\mathrm{Au}+\mathrm{Au}$ collisions at $100+100 \mathrm{GeV} /$ nucleon.

\section{GLAUBER MODEL}

\section{A. Participant and penetrating nucleons in $A-B$ collisions}

The participant nucleon number in a nucleus-nucleus collision can be estimated by using the Glauber model [13]. To do this, let's first consider a $h$ - $A$ collision. At high energy, one can treat this problem as a collision of $h$ with a tube in the nucleus (see Fig.1). Here, we assume that the cross section of the tube is given by the nucleon-nucleon inelastic cross section $\sigma_{i n} \approx 32 \mathrm{mb}$. The probability that an inelastic interaction occurs in a volume element $\sigma_{i n} \delta z$ is

$$
\delta P\left(\boldsymbol{b}, z_{n}\right)=\sigma_{i n} \delta z \rho\left(\boldsymbol{b}, z_{n}\right)=\sigma_{i n} \frac{L}{N} \rho\left(\boldsymbol{b}, z_{n}\right) \approx \frac{\sigma_{i n}}{N} \int d z \rho(\boldsymbol{b}, z)
$$

where $\rho(\boldsymbol{b}, z)$ is the nucleon density in the nucleus and $N$ is the number of equally divided volume elements in the tube (see Fig.1). The probability that $h$ interacts with $m$ elements in the tube is

$$
P(m ; \boldsymbol{b})={ }_{N} C_{m}[\delta P(\boldsymbol{b})]^{m}[1-\delta P(\boldsymbol{b})]^{N-m} .
$$

Hence, we obtain for the total probability of occurrence of inelastic reactions in a $h-A$ collision

$$
P_{\text {inel }}=\sum_{m=1}{ }_{N} C_{m}[\delta P(\boldsymbol{b})]^{m}[1-\delta P(\boldsymbol{b})]^{N-m}=1-\left[1-\frac{\sigma_{i n}}{N} T(\boldsymbol{b})\right]^{N}
$$

where $T(\boldsymbol{b})$ is the so-called thickness function which is defined as $T(\boldsymbol{b}) \equiv \int d z \rho(\boldsymbol{b}, z)$. In the continuum limit, $N \rightarrow \infty$, this probability can be written as

$$
P_{\text {inel }}(\boldsymbol{b})=1-\exp \left[-\sigma_{\text {in }} T(\boldsymbol{b})\right] .
$$

As a natural extension of the $h$-tube collision, one can regard the nucleus-nucleus collision as a superposition of tube-tube collisions. Therefore, the total number of participant nucleons and penetrating nucleons originated from each incident nucleus is given respectively by

$$
\begin{aligned}
& N_{\text {part }}(\boldsymbol{b})=\int d \boldsymbol{b}^{\prime} T\left(\boldsymbol{b}^{\prime}\right)\left\{1-\exp \left[-\sigma_{i n} T\left(\boldsymbol{b}^{\prime}+\boldsymbol{b}\right)\right]\right\}, \\
& N_{\text {pen }}(\boldsymbol{b})=\int d \boldsymbol{b}^{\prime} T\left(\boldsymbol{b}^{\prime}\right) \exp \left[-\sigma_{i n} T\left(\boldsymbol{b}^{\prime}+\boldsymbol{b}\right)\right] .
\end{aligned}
$$

Assuming the nucleon density in the Woods-Saxon form

$$
\rho(\boldsymbol{b}, z)=\frac{\rho_{0}}{1+\exp \left[\left(\sqrt{z^{2}+\boldsymbol{b}^{2}}-R\right) / d\right]},
$$

where $d=0.54 \mathrm{fm}, R_{A}=r_{0} \times A^{1 / 3}$ and $r_{0}=1.2 \mathrm{fm}$, the penetrating nucleon number in each hemisphere in $\mathrm{Pb}+\mathrm{Pb}$ collisions turns out to be 30.7 and 58.4 for the impact parameter $b \leq 3.5 \mathrm{fm}$ (centrality $5 \%$ ) and $b \leq 6.1 \mathrm{fm}$ (centrality $15 \%$ ), respectively. This means that the total number of participant nucleons in each case is $N^{\text {part }}=353.1$ and $N^{\text {part }}=297.7$, respectively. See also Table 1 . It is gratifying to see that, when compared with the data (see $N_{B-\bar{B}}$ in the next section), these numbers are almost exact.

\footnotetext{
${ }^{1}$ In a previous work by Barz et al. [9], it is not clear the role of the incident nuclei and penetrating systems.
} 


\section{B. Transverse profile of the nucleon number density}

In the present work, we are mostly interested in central collisions. So, we shall assume a cylindrically symmetrical distribution both of charge and of fluid density. However, in comparing the results with the data, we have also to take the experimental conditions into account. It is clear that the impact parameter is not always zero, but fluctuates from event to event both in modulus and direction. In order to simplify the computation, we shall symmetrize the fluid and charge densities by considering this fluctuation by averaging over $\boldsymbol{b}$. Furthermore, we assume factorization of these densities with respect to the longitudinal and transverse variables.

Let us estimate the transverse profile of this symmetrized nucleon number density. For a given nucleus-nucleus collision, whose geometry is shown in Fig.2 (with a fixed $\boldsymbol{b}$ ), and, provided that the fluid contains nucleons which collide inelastically at least once, its nucleon density projected into the transverse plane $(\boldsymbol{\rho} \equiv(x, y))$ is obtained by adding

$$
T\left(\boldsymbol{\rho} \mp \frac{\boldsymbol{b}}{2}\right) \times\left[1-\exp \left[-\sigma_{i n} T\left(\boldsymbol{\rho} \pm \frac{\boldsymbol{b}}{2}\right)\right]\right] .
$$

So, by averaging over the impact parameter $\boldsymbol{b}$ of the incident nuclei

$$
T_{\text {fluid }}(\boldsymbol{\rho})=\frac{2 \int_{0}^{b_{\max }} d b b \int_{0}^{2 \pi} d \varphi T\left(\boldsymbol{\rho}-\frac{\boldsymbol{b}}{2}\right)\left[1-\exp \left[-\sigma_{i n} T\left(\boldsymbol{\rho}+\frac{\boldsymbol{b}}{2}\right)\right]\right]}{\int_{0}^{b_{\max }} d b b \int_{0}^{2 \pi} d \varphi} .
$$

We also have, for the penetrating system and incident nuclei,

$$
\begin{aligned}
& T_{\text {pen }}(\boldsymbol{\rho})=\frac{2 \int_{0}^{b_{\max }} d b b \int_{0}^{2 \pi} d \varphi T\left(\boldsymbol{\rho}-\frac{\boldsymbol{b}}{2}\right)\left[\exp \left[-\sigma_{i n} T\left(\boldsymbol{\rho}+\frac{\boldsymbol{b}}{2}\right)\right]\right]}{\int_{0}^{b_{\max }} d b b \int_{0}^{2 \pi} d \varphi} \\
& T_{n u c l}(\boldsymbol{\rho})=\frac{2 \int_{0}^{b_{\max }} d b b \int_{0}^{2 \pi} d \varphi T\left(\boldsymbol{\rho}-\frac{\boldsymbol{b}}{2}\right)}{\int_{0}^{b_{\max }} d b b \int_{0}^{2 \pi} d \varphi} .
\end{aligned}
$$

Observe that these quantities are related by

$$
T_{\text {fluid }}(\boldsymbol{\rho})+T_{\text {pen }}(\boldsymbol{\rho})=T_{\text {nucl }}(\boldsymbol{\rho})
$$

being the integral of the right-hand side equal to $2 A$. They are shown in Fig.3. We can also define the normalized densities

$$
\tilde{T}_{i}(\boldsymbol{\rho}) \equiv \frac{T_{i}(\boldsymbol{\rho})}{\int d \rho \rho \int d \varphi T_{i}(\boldsymbol{\rho})}
$$

for the fluid, penetrating system and incident nuclei. In the following sections, we assume that the charge distribution in each system is proportional to these quantities. Also, the fluid density will be taken proportional to $\tilde{T}_{f l u i d}(\boldsymbol{\rho})$.

\section{NET-CHARGE DISTRIBUTION IN THE FLUID}

\section{A. Space-time evolution of the fluid}

As mentioned in the Introduction, we approximate the evolution of the charged matter by a longitudinally expanding scaling solution, neglecting the transverse expansion. The transverse structure has already been discussed in the previous section. In such a case, the rapidity of the fluid is given by 


$$
\eta=\frac{1}{2} \ln \frac{t+z}{t-z}
$$

It is convenient to take this and the proper time

$$
\tau=\sqrt{t^{2}-z^{2}}
$$

together with $\boldsymbol{\rho}$ as the coordinates for describing the motion of our charged matter. Thus,

$$
t=\tau \cosh \eta, \quad z=\tau \sinh \eta,
$$

for the time and the longitudinal coordinate in the center-of-mass frame. Strictly speaking, the scaling solution is only valid if one has an "infinitely long" fluid $(-\infty \leq \eta \leq+\infty)$ with an infinitely large size and constant density in the transverse directions. For a real finite fluid, transverse expansion necessarily appears and also the longitudinal form suffers degradation beginning from the edges. In the present paper, we will neglect these effects, for the sake of simplicity because we find that they are not essential as far as the central region of the collision is concerned.

\section{B. Longitudinal profile of the charge distribution}

Let us now discuss the longitudinal structure of the charge distribution. The net charge distribution in the $\eta$-variable (integrated charge distribution over $\boldsymbol{\rho}$-space) in the fluid can be estimated in terms of the rapidity distributions of the produced particles

$$
\frac{d N_{n e t}}{d y} \approx \frac{d N_{p}}{d y}-\frac{d N_{\bar{p}}}{d y}+\frac{d N_{\pi^{+}}}{d y}-\frac{d N_{\pi^{-}}}{d y}+\frac{d N_{K^{+}}}{d y}-\frac{d N_{K^{-}}}{d y}
$$

It should be noted here that the net charge distribution which we need to know is the one inside the fluid, so we have to estimate $d N_{n e t} / d y$ soon after the freeze-out, i.e., $\tau=10^{1} \sim 10^{2} \mathrm{fm}$. In this stage (before the hyperon decays), the multiplicity of the negative mesons $\left(h^{-} \equiv \pi^{-}, K^{-}\right)$is slightly larger than that of the positive mesons $\left(h^{+} \equiv \pi^{+}, K^{+}\right)$ due to the large neutron-proton asymmetry in large stable nuclei whereas no such Coulomb-energy effect is expected in the expanding system.

Consider the charge and the baryon number conservations in the collision:

$$
\begin{aligned}
N_{p}^{\text {part }}+N_{n}^{\text {part }} & =\int d y \frac{d N_{p-\bar{p}}}{d y}+\int d y \frac{d N_{n-\bar{n}}}{d y}+\int d y \frac{d N_{Y^{+}-\overline{Y^{+}}}}{d y}+\int d y \frac{d N_{Y^{0}-\overline{Y^{0}}}}{d y}+\int d y \frac{d N_{Y^{-}-\overline{Y^{-}}}}{d y} \\
N_{p}^{\text {part }} & =\int d y \frac{d N_{p-\bar{p}}}{d y}+\int d y \frac{d N_{Y^{+-}-\overline{Y^{+}}}}{d y}-\int d y \frac{d N_{Y^{-}-\overline{Y^{-}}}}{d y}-\int d y\left[\frac{d N_{h^{-}}}{d y}-\frac{d N_{h^{+}}}{d y}\right]_{\text {direct }},
\end{aligned}
$$

where $N_{p}^{\text {part }}$ and $N_{n}^{\text {part }}$ are the numbers of participant protons and neutrons, respectively, and $Y^{+}, Y^{0}$ and $Y^{-}$stand for positive, neutral and negative hyperons. For the present, we shall concentrate on estimating the net charge distribution in the case of $158 \mathrm{GeV} /$ nucleon $\mathrm{Pb}+\mathrm{Pb}$ collision for the centrality $5 \%$. From these two equations, we obtain the following formula for the negative charge excess in mesons:

$$
\begin{aligned}
\int d y\left[\frac{d N_{h^{-}}}{d y}-\frac{d N_{h^{+}}}{d y}\right] & =N_{n}^{\text {part }}-\int d y \frac{d N_{n-\bar{n}}}{d y}-\int d y \frac{d N_{\Lambda-\bar{\Lambda}}}{d y}-\int d y \frac{d N_{\Sigma^{0}-\overline{\Sigma^{0}}}}{d y}-2 \int d y \frac{d N_{\Sigma^{-}-\Sigma^{-}}}{d y} \\
& \approx \frac{A-Z}{A} \times N_{B-\bar{B}}-\left\{1.07 \times N_{p-\bar{p}}+1.6 \times N_{\Lambda-\bar{\Lambda}}\right\}
\end{aligned}
$$

Here, we used the experimental results, $N_{n-\bar{n}} / N_{p-\bar{p}} \approx 1.07, N_{\Sigma^{ \pm}-\Sigma^{ \pm}} / N_{\Lambda-\bar{\Lambda}} \approx 0.3$ [14, 15. In the last term above, $N_{\Lambda-\bar{\Lambda}}$ contains also $\Sigma^{0}-\overline{\Sigma^{0}}$. One can then estimate the negative charge excess using the experimental value for the net baryon number $N_{B-\bar{B}}=352 \pm 12$, being the net proton number $N_{p-\bar{p}}=148.9$ (See Fig.4) and $N_{\Lambda-\bar{\Lambda}}=27.4$ 15], as

$$
N_{h^{-}-h^{+}} \equiv \int d y\left[\frac{d N_{h^{-}}}{d y}-\frac{d N_{h^{+}}}{d y}\right]_{\text {direct }} \approx 10.0 .
$$

One can verify the consistency of this estimate, by computing the participant proton number $N_{p}^{\text {part }}$ which is approximately $Z / A \times N_{B-\bar{B}} \approx 138.7$ and comparing with the total net charge, obtained by using $N_{p-\bar{p}}$ data and eq. (17): $N_{p-\bar{p}}-N_{h^{-}-h^{+}} \approx 138.9$. 
Hence, by putting eq. (17) into eq. (15) and by considering that the existing negative particle data include also $\bar{p}$, finally we arrive at the following expression for the charge distribution of particles at the freeze-out time

$$
\begin{aligned}
\frac{d N_{n e t}}{d y} & \approx \frac{d N_{p}}{d y}-\frac{d N_{\bar{p}}}{d y}+\left\{\frac{d N_{\pi^{+}}}{d y}+\frac{d N_{K^{+}}}{d y}\right\}-\left\{\frac{d N_{\pi^{-}}}{d y}+\frac{d N_{K^{-}}}{d y}\right\} \\
& \approx \frac{d N_{p-\bar{p}}}{d y}-\frac{N_{h^{-}-h^{+}}}{\left\langle N_{h^{-}+\bar{p}}\right\rangle-\left\langle N_{\bar{p}}\right\rangle} \times\left\{\frac{d N_{h^{-}+\bar{p}}}{d y}-\frac{d N_{\bar{p}}}{d y}\right\} .
\end{aligned}
$$

For $\mathrm{Pb}+\mathrm{Pb}$ at $158 \mathrm{GeV} /$ nucleon, we have $N_{h^{-}+\bar{p}}=695 \pm 30$ [15] (see Fig.5). The $N_{\bar{p}}$ is estimated by using an empirical relation $\bar{p} / \Lambda=0.12 \sim 0.13$ found in $S+S$ and $S+A g$ collisions at $200 \mathrm{GeV} /$ nucleon [16, 17]. Because the multiplicity of $\bar{\Lambda} \approx 7.5(\bar{\Lambda} / \Lambda \approx 0.2)[18]$, we obtain $N_{\Lambda} \approx 35$ and $N_{\bar{p}} \approx 4.4$ (See Fig.6). The solid line in Fig.7 shows the net charge distribution obtained by eq.(18), for the centrality $5 \%$, in $158 \mathrm{GeV} /$ nucleon $\mathrm{Pb}+\mathrm{Pb}$.

Now, the rapidity distribution of produced particles given by eq.(18) is a convolution of the charge rapidity distribution in the fluid and the thermal rapidity distribution of the produced particles

$$
\frac{d N_{n e t}}{d y} \simeq \mathcal{N} \int d \eta \frac{d N_{n e t}}{d \eta} \times \frac{1}{e^{\left\langle m_{\mathrm{T}}\right\rangle \cosh (y-\eta) / T}-1},
$$

where $T=140 \mathrm{MeV}$ is the freeze-out temperature and $\left\langle m_{\mathrm{T}}\right\rangle$ is the pion mean transverse mass, evaluated with the Bose distribution at the temperature $T$. So, in computing the electromagnetic field in the following section, we took a piece-wise flat rapidity distribution for the fluid net charge as seen in Fig.7 which, upon convolution eq.(19), approximates as closely as possible the particle distribution of eq.(18). The normalization constant $\mathcal{N}$ in eq.(19) is fixed by equating the integrals of $d N_{n e t} / d y$ and $d N_{n e t} / d \eta$. As for the fluid density, as mentioned in the beginning of this section we took a completely flat $\eta$-distribution which gives the correct number of pions.

\section{Centrality dependence of the net charge distribution}

As is easily understood intuitively, the net charge distribution strongly depends on the centrality of the collision. In the previous subsection, we have estimated the net charge distribution by using the NA49 data on $158 \mathrm{GeV} /$ nucleon $\mathrm{Pb}+\mathrm{Pb}$ collision with the centrality $5 \%$ [15]. We would like to study the Coulomb effect on the $\pi^{+} / \pi^{-}$ratio, which has been measured by the NA44 Collaboration [12], considering events with $15 \%$ centrality. The question is that there are no corresponding data which would allow us to estimate the net charge distribution under the same experimental conditions. We have mentioned in Section II and verified in Section III that the Glauber model we described there gives a very good account of the NA49 data. In view of this, we will account for the centrality dependence of the net charge distribution, by the use of the referred model. Concretely, by assuming that the longitudinal structure of the net charge distribution remains the same, changing only the transverse profile and consequently the normalization, we introduce the following factor to relating quantities with different centrality $(\chi)$ :

$$
\left.\frac{d N_{h}}{d \eta}\right|_{\text {centrality } \chi^{\prime}}=\left.\frac{G\left(\chi^{\prime}\right)}{G(\chi)} \frac{d N_{h}}{d \eta}\right|_{\text {centrality } \chi},
$$

for $h=h^{-}+\bar{p}$ and $p-\bar{p}$, where $G(\chi)=N^{\text {part }}$ for the centrality $\chi$. The participant nucleon-number dependence of hyperon multiplicity is studied in Ref. [19]. Using their results, we have

$$
\left.\frac{d N_{Y}}{d \eta}\right|_{\text {centrality } \chi^{\prime}}=\left.\left[\frac{G\left(\chi^{\prime}\right)}{G(\chi)}\right]^{1.30} \frac{d N_{Y}}{d \eta}\right|_{\text {centrality } \chi},
$$

for $Y=\Lambda$ hyperon. The multiplicity values estimated for the centrality $15 \%$, by using the data obtained for the centrality $5 \%$, are summarized in Table $\mathbb{1}$.

\section{ELECTROMAGNETIC FIELDS IN HIGH-ENERGY HEAVY-ION COLLISIONS}

Having been defined the charge distributions and their time development in the previous sections, now we can compute the electromagnetic field produced by each one of such distributions. 


\section{A. Potentials of the longitudinal expanding charged matter}

An infinitesimal charge $d Q$ moving along a trajectory $\boldsymbol{r}=\boldsymbol{r}_{0}(t)$ in the hadronic or quark matter (fluid) produces a Liénard-Wiechert four-vector potential

$$
A^{\mu}(t, \boldsymbol{x})=\left(\frac{d Q}{\left|\boldsymbol{x}-\boldsymbol{r}_{0}\left(t^{\prime}\right)\right|-\boldsymbol{v}\left(t^{\prime}\right) \cdot\left\{\boldsymbol{x}-\boldsymbol{r}_{0}\left(t^{\prime}\right)\right\}}, \frac{\boldsymbol{v} d Q}{\left|\boldsymbol{x}-\boldsymbol{r}_{0}\left(t^{\prime}\right)\right|-\boldsymbol{v}\left(t^{\prime}\right) \cdot\left\{\boldsymbol{x}-\boldsymbol{r}_{0}\left(t^{\prime}\right)\right\}}\right),
$$

where $t^{\prime}$ is the retarded time satisfying the condition,

$$
t^{\prime}+\left|\boldsymbol{x}-\boldsymbol{r}_{0}\left(t^{\prime}\right)\right|=t
$$

In terms of the coordinates $(\tau, \boldsymbol{\rho}, \eta)$, for $\boldsymbol{r}_{0}\left(t^{\prime}\right)=\left(\boldsymbol{\rho}^{\prime}, v_{z} t^{\prime}\right) \equiv\left(\boldsymbol{\rho}^{\prime}, \tau^{\prime} \sinh \eta^{\prime}\right)$, eqs. (20) and (21) may be rewritten

$$
A^{\mu}(\tau, \boldsymbol{\rho}, \eta)=\left(\frac{\cosh \eta^{\prime} d Q}{\tau \cosh \left(\eta-\eta^{\prime}\right)-\tau^{\prime}}, 0,0, \frac{\sinh \eta^{\prime} d Q}{\tau \cosh \left(\eta-\eta^{\prime}\right)-\tau^{\prime}}\right)
$$

where

$$
\tau^{\prime 2}-2 \tau\left[\cosh \left(\eta-\eta^{\prime}\right)\right] \tau^{\prime}+\tau^{2}-\left|\boldsymbol{\rho}-\boldsymbol{\rho}^{\prime}\right|^{2}=0 .
$$

By solving eq.(23) with the condition $\tau \geq \tau^{\prime}$, we have

$$
\tau^{\prime}=\tau \cosh \left(\eta-\eta^{\prime}\right)-\sqrt{\tau^{2} \sinh ^{2}\left(\eta-\eta^{\prime}\right)+\left|\boldsymbol{\rho}-\boldsymbol{\rho}^{\prime}\right|^{2}}
$$

with $\tau \geq\left|\boldsymbol{\rho}-\boldsymbol{\rho}^{\prime}\right|$.

As discussed in the previous sections, the charge distribution in the expanding fluid is written as

$$
\frac{d Q\left(\eta^{\prime}, \boldsymbol{\rho}^{\prime}\right)}{d \boldsymbol{\rho}^{\prime} d \eta^{\prime}}=e \tilde{T}_{\text {fluid }}\left(\boldsymbol{\rho}^{\prime}\right) \frac{d N_{n e t}}{d \eta^{\prime}}
$$

so the total potentials due to the whole fluid, $A^{0} \equiv \phi_{\text {fluid }}(\tau, \boldsymbol{\rho}, \eta)$ and $A^{3} \equiv A_{\text {fluid }}(\tau, \boldsymbol{\rho}, \eta)$, are given by

$$
\phi_{\text {fluid }}(\tau, \boldsymbol{\rho}, \eta)=e \int d \boldsymbol{\rho}^{\prime} \tilde{T}_{f l u i d}\left(\boldsymbol{\rho}^{\prime}\right) \int d \eta^{\prime} \frac{d N_{n e t}}{d \eta^{\prime}} \frac{\cosh \eta^{\prime} \times \theta\left(\tau-\left|\boldsymbol{\rho}-\boldsymbol{\rho}^{\prime}\right|\right)}{\sqrt{\tau^{2} \sinh ^{2}\left(\eta-\eta^{\prime}\right)+\left|\boldsymbol{\rho}-\boldsymbol{\rho}^{\prime}\right|^{2}}}
$$

and

$$
A_{\text {fluid }}(\tau, \boldsymbol{\rho}, \eta)=e \int d \boldsymbol{\rho}^{\prime} \tilde{T}_{f l u i d}\left(\boldsymbol{\rho}^{\prime}\right) \int d \eta^{\prime} \frac{d N_{\text {net }}}{d \eta^{\prime}} \frac{\sinh \eta^{\prime} \times \theta\left(\tau-\left|\boldsymbol{\rho}-\boldsymbol{\rho}^{\prime}\right|\right)}{\sqrt{\tau^{2} \sinh ^{2}\left(\eta-\eta^{\prime}\right)+\left|\boldsymbol{\rho}-\boldsymbol{\rho}^{\prime}\right|^{2}}}
$$

respectively.

\section{B. Potentials of the incident nuclei and the penetrating nuclear systems}

The incident nuclei ( $n u c l$ ) and the penetrating nuclear systems which are not included in the fluid (pen) also produce electromagnetic fields. Here, we assume that the penetrating systems consist of nucleons which suffered no inelastic collision and take their rapidities $y$ the same as the beam rapidities $\pm y_{b e}$. The scalar and vector potentials can be obtained in a similar way as in the case of fluid. The potentials due to the incident nuclei are

$$
\phi_{\text {nucl }}(\tau, \boldsymbol{\rho}, \eta)=e Z \int d \boldsymbol{\rho}^{\prime} \tilde{T}_{n u c l}\left(\boldsymbol{\rho}^{\prime}\right) \sum_{\eta^{\prime}= \pm y_{b e}}\left[\frac{\cosh \eta^{\prime} \times \theta\left(\left|\boldsymbol{\rho}-\boldsymbol{\rho}^{\prime}\right|-\tau\right)}{\sqrt{\tau^{2} \sinh ^{2}\left(\eta-\eta^{\prime}\right)+\left|\boldsymbol{\rho}-\boldsymbol{\rho}^{\prime}\right|^{2}}}\right]
$$

and

$$
A_{n u c l}(\tau, \boldsymbol{\rho}, \eta)=e Z \int d \boldsymbol{\rho}^{\prime} \tilde{T}_{n u c l}\left(\boldsymbol{\rho}^{\prime}\right) \sum_{\eta^{\prime}= \pm y_{b e}}\left[\frac{\sinh \eta^{\prime} \times \theta\left(\left|\boldsymbol{\rho}-\boldsymbol{\rho}^{\prime}\right|-\tau\right)}{\sqrt{\tau^{2} \sinh ^{2}\left(\eta-\eta^{\prime}\right)+\left|\boldsymbol{\rho}-\boldsymbol{\rho}^{\prime}\right|^{2}}}\right]
$$


where $Z$ is the proton number of each of the incident nuclei and $\tilde{T}_{n u c l}\left(\boldsymbol{\rho}^{\prime}\right)$ is the renormalized thickness function defined in eq.(11). For the penetrating systems, we have

$$
\phi_{p e n}(\tau, \boldsymbol{\rho}, \eta)=e\left\langle Z^{\prime}\right\rangle \int d \boldsymbol{\rho}^{\prime} \tilde{T}_{p e n}\left(\boldsymbol{\rho}^{\prime}\right) \sum_{\eta^{\prime}= \pm y_{b e}}\left[\frac{\cosh \eta^{\prime} \times \theta\left(\tau-\left|\boldsymbol{\rho}-\boldsymbol{\rho}^{\prime}\right|\right)}{\sqrt{\tau^{2} \sinh ^{2}\left(\eta-\eta^{\prime}\right)+\left|\boldsymbol{\rho}-\boldsymbol{\rho}^{\prime}\right|^{2}}}\right]
$$

and

$$
A_{p e n}(\tau, \boldsymbol{\rho}, \eta)=e\left\langle Z^{\prime}\right\rangle \int d \boldsymbol{\rho}^{\prime} \tilde{T}_{p e n}\left(\boldsymbol{\rho}^{\prime}\right) \sum_{\eta^{\prime}= \pm y_{b e}}\left[\frac{\sinh \eta^{\prime} \times \theta\left(\tau-\left|\boldsymbol{\rho}-\boldsymbol{\rho}^{\prime}\right|\right)}{\sqrt{\tau^{2} \sinh ^{2}\left(\eta-\eta^{\prime}\right)+\left|\boldsymbol{\rho}-\boldsymbol{\rho}^{\prime}\right|^{2}}}\right]
$$

where $\left\langle Z^{\prime}\right\rangle$ is the average number of protons which are included in each penetrating system and $\tilde{T}_{\text {pen }}\left(\boldsymbol{\rho}^{\prime}\right)$ is the renormalized thickness function defined in eq.(11).

\section{Total electromagnetic field in high-energy heavy-ion collisions}

The total scalar and vector potentials produced in high-energy heavy-ion collisions are now written as the following sums:

$$
\begin{aligned}
& \phi(\tau, \boldsymbol{\rho}, \eta)=\phi_{\text {fluid }}(\tau, \boldsymbol{\rho}, \eta)+\phi_{\text {nucl }}(\tau, \boldsymbol{\rho}, \eta)+\phi_{\text {pen }}(\tau, \boldsymbol{\rho}, \eta), \\
& A_{z}(\tau, \boldsymbol{\rho}, \eta)=A_{\text {fluid }}(\tau, \boldsymbol{\rho}, \eta)+A_{\text {nucl }}(\tau, \boldsymbol{\rho}, \eta)+A_{\text {pen }}(\tau, \boldsymbol{\rho}, \eta) .
\end{aligned}
$$

Then, it is straightforward to obtain the corresponding electromagnetic fields by

$$
\begin{aligned}
& \boldsymbol{E}(\boldsymbol{x}, t)=-\boldsymbol{\nabla} \phi(\boldsymbol{x}, t)-\frac{\partial}{\partial t} \boldsymbol{A}(\boldsymbol{x}, t), \\
& \boldsymbol{B}(\boldsymbol{x}, t)=\boldsymbol{\nabla} \times \boldsymbol{A}(\boldsymbol{x}, t)
\end{aligned}
$$

and they are explicitly given by

$$
\begin{aligned}
& E_{x}(\tau, \boldsymbol{\rho}, \eta)=\int d \boldsymbol{\rho}^{\prime} \int d \eta^{\prime} \frac{\mathcal{F}\left(\eta^{\prime}, \boldsymbol{\rho}^{\prime}\right) \cosh \eta^{\prime}}{\left[\tau^{2} \sinh ^{2}\left(\eta-\eta^{\prime}\right)+\left|\boldsymbol{\rho}-\boldsymbol{\rho}^{\prime}\right|^{2}\right]^{3 / 2}} \times\left(\boldsymbol{\rho}-\boldsymbol{\rho}^{\prime}\right)_{x} \\
& E_{y}(\tau, \boldsymbol{\rho}, \eta)=\int d \boldsymbol{\rho}^{\prime} \int d \eta^{\prime} \frac{\mathcal{F}\left(\eta^{\prime}, \boldsymbol{\rho}^{\prime}\right) \cosh \eta^{\prime}}{\left[\tau^{2} \sinh ^{2}\left(\eta-\eta^{\prime}\right)+\left|\boldsymbol{\rho}-\boldsymbol{\rho}^{\prime}\right|^{2}\right]^{3 / 2}} \times\left(\boldsymbol{\rho}-\boldsymbol{\rho}^{\prime}\right)_{y} \\
& E_{z}(\tau, \boldsymbol{\rho}, \eta)=\int d \boldsymbol{\rho}^{\prime} \int d \eta^{\prime} \frac{\mathcal{F}\left(\eta^{\prime}, \boldsymbol{\rho}^{\prime}\right) \sinh \left(\eta-\eta^{\prime}\right)}{\left[\tau^{2} \sinh ^{2}\left(\eta-\eta^{\prime}\right)+\left|\boldsymbol{\rho}-\boldsymbol{\rho}^{\prime}\right|^{2}\right]^{3 / 2}} \times \tau \\
& B_{x}(\tau, \boldsymbol{\rho}, \eta)=\int d \boldsymbol{\rho}^{\prime} \int d \eta^{\prime} \frac{-\mathcal{F}\left(\eta^{\prime}, \boldsymbol{\rho}^{\prime}\right) \sinh \eta^{\prime}}{\left[\tau^{2} \sinh ^{2}\left(\eta-\eta^{\prime}\right)+\left|\boldsymbol{\rho}-\boldsymbol{\rho}^{\prime}\right|^{2}\right]^{3 / 2}} \times\left(\boldsymbol{\rho}-\boldsymbol{\rho}^{\prime}\right)_{y} \\
& B_{y}(\tau, \boldsymbol{\rho}, \eta)=\int d \boldsymbol{\rho}^{\prime} \int d \eta^{\prime} \frac{\mathcal{F}\left(\eta^{\prime}, \boldsymbol{\rho}^{\prime}\right) \sinh \eta^{\prime}}{\left[\tau^{2} \sinh ^{2}\left(\eta-\eta^{\prime}\right)+\left|\boldsymbol{\rho}-\boldsymbol{\rho}^{\prime}\right|^{2}\right]^{3 / 2}} \times\left(\boldsymbol{\rho}-\boldsymbol{\rho}^{\prime}\right)_{x} \\
& B_{z}(\tau, \boldsymbol{\rho}, \eta)=0,
\end{aligned}
$$

where

$$
\begin{aligned}
\mathcal{F}\left(\eta^{\prime}, \boldsymbol{\rho}^{\prime}\right) & =e\left[\tilde{T}_{\text {fluid }}\left(\boldsymbol{\rho}^{\prime}\right) \frac{d N_{n e t}}{d \eta^{\prime}}+\left\langle Z^{\prime}\right\rangle \tilde{T}_{\text {pen }}\left(\boldsymbol{\rho}^{\prime}\right)\left\{\delta\left(\eta^{\prime}-y_{b e}\right)+\delta\left(\eta^{\prime}+y_{b e}\right)\right\}\right] \theta\left(\tau-\left|\boldsymbol{\rho}-\boldsymbol{\rho}^{\prime}\right|\right) \\
+ & e\left[Z \tilde{T}_{n u c l}\left(\boldsymbol{\rho}^{\prime}\right)\left\{\delta\left(\eta^{\prime}-y_{b e}\right)+\delta\left(\eta^{\prime}+y_{b e}\right)\right\}\right] \theta\left(\left|\boldsymbol{\rho}-\boldsymbol{\rho}^{\prime}\right|-\tau\right)
\end{aligned}
$$




\section{Space-time structure of the electromagnetic field}

In order to visualize the space-time distribution of the electromagnetic field in high-energy heavy-ion collisions, we show now some results of numerical calculations by means of eqs.(36)-(41). First, Fig.8 shows the transverse component of the electric field, $E_{\rho}\left(\equiv \sqrt{E_{x}^{2}+E_{y}^{2}}\right)$, as function of $\rho\left(\equiv \sqrt{x^{2}+y^{2}}\right)$ at $t=15$ fm in the center of mass system. One can see a high peak in the electric field which increases as $z \rightarrow t$, i.e., as the proper time $\tau \rightarrow 0$. This peak is produced by one of the incident nuclei before the collision. For smaller values of $z$, the peak which appears close to $\rho \sim R_{P b}$ (radius of $\mathrm{Pb}$ ) is produced by the expanding charged fluid. The time dependence of $E_{\rho}$ at $\rho=5$ fm for various $z$ are shown in Fig.9. One can observe the propagation of a sharp peak produced by one of the incident nuclei. The apparent discrepancy of the result for $z=0$ is due to the fact that both nuclei contribute to the field in this case. It is also found that the width of the peak is about $\Delta \tau \sim 3 \mathrm{fm}$.

The longitudinal component of the electric field $E_{z}$ is shown in Fig.10. The magnitude of $E_{z}$ is much smaller than that of $E_{\rho}$. At $z=0, E_{z} \equiv 0$ because of the symmetry. Except for the case of $z=14 \mathrm{fm}$ at small $\rho$, the sign of $E_{z}$ is negative mainly due to the charge concentration at large- $\eta$ region (see Fig. 7 ). The positive $E_{z}$ in $z=14$ fm is due to one of the incident nuclei.

The magnetic field at $t=15 \mathrm{fm}$ is shown in Fig. 11. Because of the expansion of a positively charged matter, the sign of $B_{\varphi}$ at $z>0$ is positive. One can also roughly estimate the maximum value of the electromagnetic energy density $\varepsilon_{\max }$. The maximum value of $E_{\rho} \approx 7.5[\mathrm{MeV} / \mathrm{fm}]$ is reached on $z \sim 0 \mathrm{fm}$ and $\rho \sim R_{P b}$ at $t \sim 1 \mathrm{fm}$. Then we have $\varepsilon_{\max } \approx 0.3 \mathrm{MeV} / \mathrm{fm}^{3}$.

\section{FREEZE-OUT SURFACE}

To obtain the freeze-out surface, we use the particle density in the fluid parametrized as described in Section III,

$$
n(\tau, \eta, \rho)=\tilde{T}_{\text {fluid }}(\rho) \frac{d N_{\text {fluid }}}{d \eta} \times \frac{1}{\tau},
$$

where $d N_{\text {fluid }} / d \eta$ is constant in some rapidity interval. Since the most of the produced particles are pions, let us reinterpret it as the portion which corresponds to pions and fix the parameters by fitting the data on

$$
\frac{d N_{\pi}}{d y} \approx 3 \times \frac{d N_{\pi^{-}}}{d y}
$$

where $d N_{\pi} / d y$ is related with $d N_{\text {fluid }} / d \eta$ through

$$
\frac{d N_{\pi}}{d y} \simeq \mathcal{N} \int d \eta \frac{d N_{\text {fluid }}}{d \eta} \times \frac{1}{e^{\left\langle m_{\mathrm{T}}\right\rangle \cosh (y-\eta) / T}-1} .
$$

Here we are including only pions, considering the other hadrons as contaminations. The NA49's data for negative hadrons include $\bar{p}$ and $K^{-} 15$. . Using the experimental results in the mid rapidity region, $\left\langle K^{+}+K^{-}+2 K_{s}^{0}\right\rangle /\left\langle\pi^{+}+\right.$ $\left.\pi^{0}+\pi^{-}\right\rangle \approx 0.135, K^{+} / K^{-} \approx 1.8$ and $K_{s}^{0} / K^{-} \approx 1.8[18]$, one can estimate the multiplicity of kaons as shown in Table. II and the total pion multiplicity (including $\pi^{0}$ ) is 1950 and 1640 for the centrality 5\% and 15\%, respectively. The solid line in Fig.12 shows the total pion rapidity distribution for centrality $15 \%$. The shaded area and the dashed curve are our parametrization for $d N_{\text {fluid }} / d \eta$ and $d N_{\pi} / d y$ respectively.

Now, we can compute the freeze out surface by equating the density given in eq. (43) to the critical value $n_{f}$ for the Bose-Einstein distribution at the freeze-out temperature $T_{f}$

$$
\left.\tilde{T}_{\text {fluid }}(\rho) \frac{d N_{\text {fluid }}}{d \eta}\right|_{\eta=0} \frac{1}{\tau}=n_{f} \equiv \frac{3 m_{\pi}^{2} T_{f}}{2 \pi^{2}} \sum_{n=0}^{\infty} \frac{K_{2}\left[(n+1) \frac{m_{\pi}}{T_{f}}\right]}{n+1}
$$

For $T_{f}=140 \mathrm{MeV}$, one obtains $n_{f}=0.096 \mathrm{fm}^{-3}$. The intercept of the freeze-out surface in $(\tau, \rho)$-plane is shown in Fig.13. 


\section{SINGLE-PARTICLE SPECTRA AND COULOMB EFFECT}

\section{A. Transverse-momentum spectra of charged particles}

Now that the electromagnetic field and the freeze-out surface have been obtained, one can solve the equation of motion for a classical charged particle,

$$
\frac{d}{d t} \frac{m \boldsymbol{v}(t)}{\sqrt{1-\boldsymbol{v}^{2}(t)}}=q[\boldsymbol{E}(\boldsymbol{x}, t)+\boldsymbol{v}(t) \times \boldsymbol{B}(\boldsymbol{x}, t)],
$$

starting from the freeze-out surface. In the present work, we have neglected the effect of the Coulomb field before the freeze out. The time evolution of the $\pi^{+}$- and the $\pi^{-}$- momenta, as given by eq.(47), can generally be expressed in terms of the location and the initial momentum $m_{\mathrm{T} 0}$ at the freeze out as

$$
m_{\mathrm{T}}^{ \pm}=m_{\mathrm{T}}^{ \pm}\left(\rho, \eta ; m_{\mathrm{T} 0}, y_{0}, \varphi\right)
$$

where $\rho, \eta, m_{\mathrm{T} 0}, y_{0}, \varphi$ are the phase space coordinates of $\pi$ at the freeze-out time. We show in Fig.14 some results for the specified initial conditions. One can see that $\pi^{+} \mathrm{s}$ are accelerated outward and $\pi^{-} \mathrm{s}$ are decelerated by the electromagnetic field. The rapidity $y_{0}$ also shifts to $y_{ \pm}$. However, this change is quite small, $\left|y_{ \pm}-y_{0}\right| \ll 0.1$.

Since the particle number density is given by eq.(43), if we neglect the Coulomb effect, the invariant cross section for $\pi^{ \pm, 0}$ would be written in the form

$$
\begin{aligned}
E \frac{1}{\sigma_{\text {tot }}} \frac{d^{3} \sigma}{d^{3} \boldsymbol{p}} & =\frac{1}{(2 \pi)^{3}} \iiint \int_{F . \text { O.surface }} p_{\mu} d \sigma^{\mu} \frac{1}{e^{m_{T 0} \cosh (\eta-y) / T_{f}}-1} \\
& =\frac{1}{(2 \pi)^{3}} \int d \phi \int_{\eta_{\min }}^{\eta_{\max }} d \eta\left\{\int \rho d \rho \tau_{f}(\rho) m_{\mathrm{To}} \cosh (\eta-y)+\int d \tau \tau \rho_{f}(\tau) p_{\mathrm{T} 0} \cos (\varphi-\phi)\right\} \frac{1}{e^{m_{T 0} \cosh (\eta-y) / T_{f}}-1}
\end{aligned}
$$

So, the transverse momentum distribution in this case is

$$
\frac{d N}{m_{\mathrm{T} 0} d m_{\mathrm{T} 0}}=\frac{1}{(2 \pi)^{2}} \iint d y d \varphi \int_{\eta_{\min }}^{\eta_{\max }} d \eta\left\{\int \rho d \rho \tau_{f}(\rho) m_{\mathrm{T} 0} \cosh (\eta-y)+\int d \tau \tau \rho_{f}(\tau) p_{\mathrm{T} 0} \cos \varphi\right\} \frac{1}{e^{m_{T 0} \cosh (\eta-y) / T_{f}}-1} .
$$

Since the transverse mass $m_{\mathrm{T} 0}$ at freeze-out time shifts to $m_{\mathrm{T}}^{ \pm}$as given by eq.(48), the single particle spectrum eq. (50) is distorted for $\pi^{+}$as

$$
\begin{aligned}
\frac{d N^{+}}{m_{\mathrm{T}}^{+} d m_{\mathrm{T}}^{+}}= & \frac{1}{(2 \pi)^{2}} \iint d y d \varphi \int_{\eta_{\min }}^{\eta_{\max }} d \eta \int \rho d \rho \tau_{f}(\rho) \\
& \times \int d m_{\mathrm{T} 0} \delta\left[m_{\mathrm{T} 0}-m_{\mathrm{T}}^{+}\left(\rho, \eta, m_{\mathrm{T}}, y, \varphi\right)\right]\left\{m_{\mathrm{T} 0} \cosh (\eta-y)+p_{\mathrm{T} 0}\left|\frac{\partial \tau_{f}(\rho)}{\partial \rho}\right| \cos \varphi\right\} \frac{1}{e^{m_{\mathrm{T} 0} \cosh (\eta-y) / T_{f}}-1} \\
= & \frac{1}{(2 \pi)^{2}} \iint d y d \varphi \int_{\eta_{\min }}^{\eta_{\max }} d \eta \rho \rho \rho \tau_{f}(\rho)\left|\frac{\partial m_{\mathrm{T} 0}}{\partial m_{\mathrm{T}}^{+}}\right|\left\{m_{m_{\mathrm{T} 0}=m_{\mathrm{T}}^{+}}^{+} \cosh (\eta-y)+p_{\mathrm{T}}^{+}\left|\frac{\partial \tau_{f}(\rho)}{\partial \rho}\right| \cos \varphi\right\} \frac{1}{e^{m_{\mathrm{T}}^{+}} \cosh (\eta-y) / T_{f}}-1
\end{aligned}
$$

For $\pi^{-}$, one can obtain a similar expression.

However, these are not yet the final results for the charged-particle spectra. In writing eq.(51), we have neglected the possibility of frozen-out particles be reabsorbed by the fluid again. So, we have checked all the trajectories of $\pi^{ \pm}$mesons after leaving the freeze-out surface and excluded those ones which crossed it in, considering them as re-absorbed by the fluid.

\section{B. Yield ratio $\pi^{-} / \pi^{+}$}

Using the final results of eq.(51) which we have just derived, we obtain a formula for the pionic yield ratio

$$
\pi^{-} / \pi^{+}=\frac{1}{m_{\mathrm{T}}^{-}} \frac{d N^{-}}{d m_{\mathrm{T}}^{-}} /\left.\frac{1}{m_{\mathrm{T}}^{+}} \frac{d N^{+}}{d m_{\mathrm{T}}^{+}}\right|_{m_{\mathrm{T}}^{-}=m_{\mathrm{T}}^{+} \equiv m_{\mathrm{T}}} .
$$


To compare more precisely our results with the NA44 data [12] for centrality $15 \%$, we introduce the acceptance correction factor $\mathcal{A}\left(m_{\mathrm{T}}, y\right)$ to our formula eq.(51), which would approximately reproduce the nature of the NA44's detectors:

$$
\mathcal{A}\left(m_{\mathrm{T}} ; y\right)=\delta\left(y+1.67 \sqrt{m_{\mathrm{T}}^{2}-m_{\pi}^{2}}-4.2\right) .
$$

Here we neglect the rapidity shift due to the Coulomb field.

The results of our calculation together with NA44's data are shown in Fig.15. As seen, our results approximately reproduce the data. We shall now look more carefully into our results in Fig.15. It is found that our model overestimate in the region $m_{\mathrm{T}}-m_{\pi} \sim 5 \mathrm{MeV}$ and underestimate in the region $10 \leq m_{\mathrm{T}}-m_{\pi} \leq 80 \mathrm{MeV}$. The underestimation of our model may be improved by introducing a transverse expansion for the charged fluid, because it makes the freeze-out time shorter and then the frozen out particles would feel a stronger electromagnetic field. Another factor which we have neglected in the present calculation, namely the effect of the field before the freeze out, also contributes to correct the results in the right direction. It has also been suggested by other authors [20] that $\pi^{-} / \pi^{+}$ratio has origin in hyperon decays 2 .

Save particle creation and annihilation, no quantum effect has been considered in the present calculation. Due to the uncertainty relation, evidently the classical calculation only has meaning if the wave packets are sufficiently small. This implies that the Coulomb effect for small- $p_{\text {T }}$ particles [8.22 would be smaller, because such particles would have a large wave packet for a good definition of the momentum and would feel a weaker field, which is an average field over their extension. This deviation would be larger for $\pi^{-}$than for $\pi^{+}$, because the former is attracted toward the symmetry axis whereas the latter is repelled away.

We also show our result of $\pi^{-} / \pi^{+}$for the centrality $5 \%$ in Fig.16. In this calculation, the rapidity of the pions is fixed at $y_{\pi}=0.0$ and 2.0 and set $\mathcal{A}\left(m_{\mathrm{T}} ; y\right) \equiv 1$.

\section{CONCLUDING REMARKS AND DISCUSSIONS}

In this paper, we have investigated the structure of the electromagnetic fields which accompany high-energy heavyion collisions and computed the Coulomb effect for pion spectra caused as a consequence. We have considered three possible origins of the total electromagnetic field, i. e., i) incident nuclei, ii) longitudinally expanding charged matter and iii) penetrating systems which are composed of surviving protons. Glauber model has been used to estimate the number of participant nucleons and to determine the transverse profile of the charged system after the collision. The centrality dependence of the net-charge is also taken into account. In the description of the space-time evolution of the charged matter, a parametrization based on one-dimensional hydrodynamical model has been used, with a constant rapidity $\eta$.

The main results of this paper could be summarized as the following.

- Compared with the expanding charged matter, the incident nuclei bring a rather strong electromagnetic field into the interaction region in $\tau \sim$ a few fm.

- The $\mathrm{NA} 44 \mathrm{~Pb}+\mathrm{Pb}$ data on $\pi^{-} / \pi^{+}$are approximately reproduced by our model (especially due to the longitudinally expanding fluid part). This means that the longitudinally expanding charged matter plays an important role on the final-state interaction of produced hadrons.

- As expected, the penetrating systems seem to be not important for the observables in the mid-rapidity region. (However, it should be noted that they could have non-negligible contributions in the fragmentation regions.)

In addition to these points, we would like to note that the Coulomb effect seen in the yield ratio $\pi^{-} / \pi^{+}$may depend both on the dumping speed of the electromagnetic field and on the cooling speed of the hadronic matter. This point leads us further into consideration of the Coulomb effect which could be observed in the RHIC energies. One would expect that the strength of Coulomb effect may be small, because the fluid takes much time to cool down before the freeze out begins and the net-charge density in mid rapidity region become small at that time.

\footnotetext{
${ }^{2}$ Although most of $\pi^{-}$from $\Lambda$-decay should appear as small $p_{\mathrm{T}}$ particles, in the NA44 Collaboration's setup almost all the hyperons (with rapidity in the range $y_{l a b .} \leq 2 \sim 3$ ) decay in the first dipole magnet region 21 . This means that very few daughter $\pi^{-}$can enter their detector. So, although we think that this effect should be studied, it is seemingly very small.
} 
To see this quantitatively, we present the prediction for the Coulomb effect in $\mathrm{Au}+\mathrm{Au}$ collisions (centrality $5 \%$ ) at $100 \mathrm{GeV}+100 \mathrm{GeV}$ energy in Fig.19. The net-charge distribution and the fluid distribution used in this calculation are shown in Fig.17 and Fig.18, respectively. For the net-charge distribution, we assume that the total net charge and width of 'shoulders' at large $|\eta|$ regions is to be the same as that of SPS case . The total number of pions in the RHIC energy in Fig.18 is determined by the energy conservation law for the total system. As shown in Fig.19, the enhancement of $\pi^{-} / \pi^{+}$is seen at very small $m_{\mathrm{T}}-m_{\pi}$ region only.

As remarked in Section VI, there remained some open questions: effect of the transverse expansion, charge redistribution inside the fluid, introduction of the resonance decays and the effect of the uncertainty relation. These questions are presently under investigation.

\section{Acknowledgments}

This work has been partially supported by FAPESP under the contracts 98/2463-2 and 98/00317-2. The authors acknowledge stimulating discussions with T. Kodama, H-T. Elze, F. Grassi and S. Padula. We are especially indebted to G. Odyniec (NA49 Collab.) and M. Kaneta (NA44 Collab.) for clarification of data and experimental set up. One of the authors (T. O.) would like to thank M. Maruyama, F. Takagi and M. Biyajima for helpful discussions and encouragement and wishes to express gratitude to M. Ueda for helpful suggestions and hospitable support at São Paulo.

[1] See, for example, M. Gyulassy, S.K. Kauffman and L.W. Wilson, Phys. Rev. C20 (1979), 2267; For recent review, G. Baym, Acta. Phys. Polon. B29 (1998), 1839.

[2] W. Benenson et al., Phys. Rev. Lett. 43 (1979), 683.

[3] K.G. Libbrecht and S.E. Koonin, Phys. Rev. Lett. 43 (1979), 1581.

[4] G. Bertsch, Nature 283 (1980), 280.

[5] M. Gyulassy and S.K. Kauffman, Nucl. Phys. A362 (1981), 503.

[6] E.V. Shuryak, Nucl. Phys. A533 (1991), 761.

[7] M.G.H. Mostafa and C-Y.Wong, Phys. Rev. C51 (1995), 2135.

[8] T. Osada, S. Sano, M. Biyajima and G. Wilk, Phys. Rev. C54 (1996), 2167R; C55 (1997), 2615.

[9] H.W. Barz, J.P. Bondorf, J.J. Gaardhoje and H. Heiselberg, Phys. Rev. C57 (1998), 2536; C56 (1997), 1553.

[10] L.D. Landau, Izv. Akd. Nauk SSSR 17, (1953) 51; L. D. Landau and S. Z. Belenkij, Usp. Phys. Nauk 56, (1956) 309; Nuovo Cimento Suppl. 3, (1956) 15; Collected Papers of L. D. Landau ed. Ter Haar (Gordon and Breach, New York, 1965).

[11] J.D. Bjorken, Phys. Rev. D27 (1983), 140.

[12] H. Bøggild et al., NA44 Collaboration, Phys. Lett. B372 (1996), 339.

[13] See, for example, Cheuk-Yin Wong, "Introduction to High-Energy Heavy-Ion Collisions", World Scientific, (1994).

[14] G. Roland for the NA49 Collabolation, Nucl. Phys. A638 (1998), 91c.; P.G. Jones, NA49 Collaboration, Nucl. Phys. A610 (1996), 188c.

[15] H. Appelshauser et al., NA49 Collaboration, nucl-ex/9810014.

[16] T. Alber et al., NA35 Collaboration, Z. Phys. C64 (1994), 195.

[17] T. Alber et al., NA35 Collaboration, Phys. Lett. B366 (1994), 56.

[18] C. Bormann et al., NA49 Collaboration, J. Phys. G23 (1997), 1817.

[19] E. Andersen et al., Phys. Lett. B433 (1998), 209.

[20] N. Arbex, U. Ornik, M. Plümer, B.R. Schlei and R.M. Weiner, Phys. Lett. B391 (1997), 465.

[21] M. Kaneta (NA44 Collaboration), private communication.

[22] T. Osada, Ph.D. Thesis, Tohoku University, 1998; T. Osada, M. Maruyama and F. Takagi, in preparation.

\footnotetext{
${ }^{3}$ We also calculated $\pi^{-} / \pi^{+}$by using a somewhat different net charge distribution, $d N_{\text {fluid }} / d \eta \sim 10$, for the central region. However, no considerable difference was obtained.
} 


\begin{tabular}{|c|c|c|c|c|}
\hline \multicolumn{5}{|c|}{$\overline{\mathrm{Pb}+\mathrm{Pb} 158 \mathrm{GeV} / \text { nucleon }}$} \\
\hline & \multicolumn{2}{|c|}{ 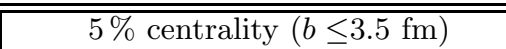 } & \multicolumn{2}{|c|}{ "15\% centrality $(b \leq 6.1 \mathrm{fm})$} \\
\hline$N^{\text {part }}$ & 352 & $N_{B-\bar{B}}$ in Ref. 15 & 298 & Glauber \\
\hline$p-\bar{p}$ & 148.9 & Ref. 15 & 125.5 & Glauber \\
\hline$h^{-} \equiv \pi^{-}, K^{-}, \bar{p}$ & 695 & Ref. 15 & 585.9 & Glauber \\
\hline$\Lambda-\bar{\Lambda}$ & 27.4 & Ref. 18 & 22.5 & Ref. 19 +Glauber \\
\hline $\bar{\Lambda}$ & 7.5 & Ref. 18 & 5.6 & Ref. 19 +Glauber \\
\hline$N_{\text {net }}=2 Z$ & 139 & & 116 & \\
\hline$<Z^{\prime}>$ & 12.5 & single hemisphere & 24.6 & single hemisphere \\
\hline$K^{+}$ & 81 & $\mid 18 \|$ & $\overline{68}$ & Glauber \\
\hline$K^{-}$ & 45 & 18 & 38 & Glauber \\
\hline
\end{tabular}

TABLE I.

\section{Table caption}

Table I: Centrality dependence of the participant nucleon numbers, hadron multiplicities and net charge excess in $\mathrm{Pb}+\mathrm{Pb}$ collision at $158 \mathrm{GeV} /$ nucleon. 


\section{Figure captions}

Fig.1: $h$-tube interaction in high-energy $h$ - $A$ collisions.

Fig.2: $A-B$ collision with impact parameter $\boldsymbol{b}$ as seen in the transverse $(x-y)$ plane.

Fig.3: Transverse profile of the average nucleon density for the fluid, penetrating systems and incident nuclei, respectively. The results are shown for $\mathrm{Pb}+\mathrm{Pb}$ collisions (centrality 5\%).

Fig.4: Net proton $(p-\bar{p})$ distribution in $\mathrm{Pb}+\mathrm{Pb} 158 \mathrm{GeV} /$ nucleon.

Fig.5: Negative hadron $\left(\pi^{-}, K^{-}\right.$and $\left.\bar{p}\right)$ distribution in $\mathrm{Pb}+\mathrm{Pb} 158 \mathrm{GeV} /$ nucleon.

Fig.6: $\bar{\Lambda}$ distribution and estimated $\bar{p}$ distribution in $\mathrm{Pb}+\mathrm{Pb} 158 \mathrm{GeV} /$ nucleon.

Fig.7: Net-charge distribution of the fluid (shaded area). The dashed curve is the result of convolution, eq.(19). The solid curve is the charged particle distribution, eq.(18).

Fig.8: Electric field $E_{\rho}$ at $t=15 \mathrm{fm}$.

Fig.9: Time dependence of $E_{\rho}$ at $\rho=5 \mathrm{fm}$.

Fig.10: Longitudinal component of the electric field $E_{z}$ at $t=15 \mathrm{fm}$.

Fig.11: Magnetic field $B_{\varphi}$ at $t=15 \mathrm{fm}$.

Fig.12: Total pion distribution and fluid distribution $d N_{\text {fluid }} / d \eta$.

Fig.13: Freeze-out surface projected on the $\tau$ - $\rho$ plane. Due to our parametrization, eq.(12), the curve in $\tau$ - $\rho$ plane is independent of $\eta$.

Fig.14: Time evolution of the momentum of $\pi^{+}$(solid line) and $\pi^{-}$(dashed line). Initial conditions are chosen as $x=6 \mathrm{fm}, y=z=0 \mathrm{fm}$ at $t=15 \mathrm{fm}$ with momentum, 20, 40, 60 and $80 \mathrm{MeV}$ in the $+x$ direction(transverse radial direction).

Fig.15: Comparison of our result for the yield ratio $\pi^{-} / \pi^{+}$(solid line) with NA44 Collab's data [12]. Remark that we did not fit the data, but the normalization of the curve has been done by estimating the overall ratio through data.

Fig.16: Our prediction of $\pi^{-} / \pi^{+}$in $\mathrm{Pb}+\mathrm{Pb} 158 \mathrm{GeV} /$ nucleon for the centrality $5 \%$. The pion rapidity $y_{\pi}$ is fixed to be 0.0 and 2.0.

Fig.17: Net charge distribution at a RHIC energy

Fig.18: Fluid distribution at a RHIC energy. The pion multiplicity is determined by the energy conservation law for the total system.

Fig.19: Prediction for $\pi^{-} / \pi^{+}$at a RHIC energy. 


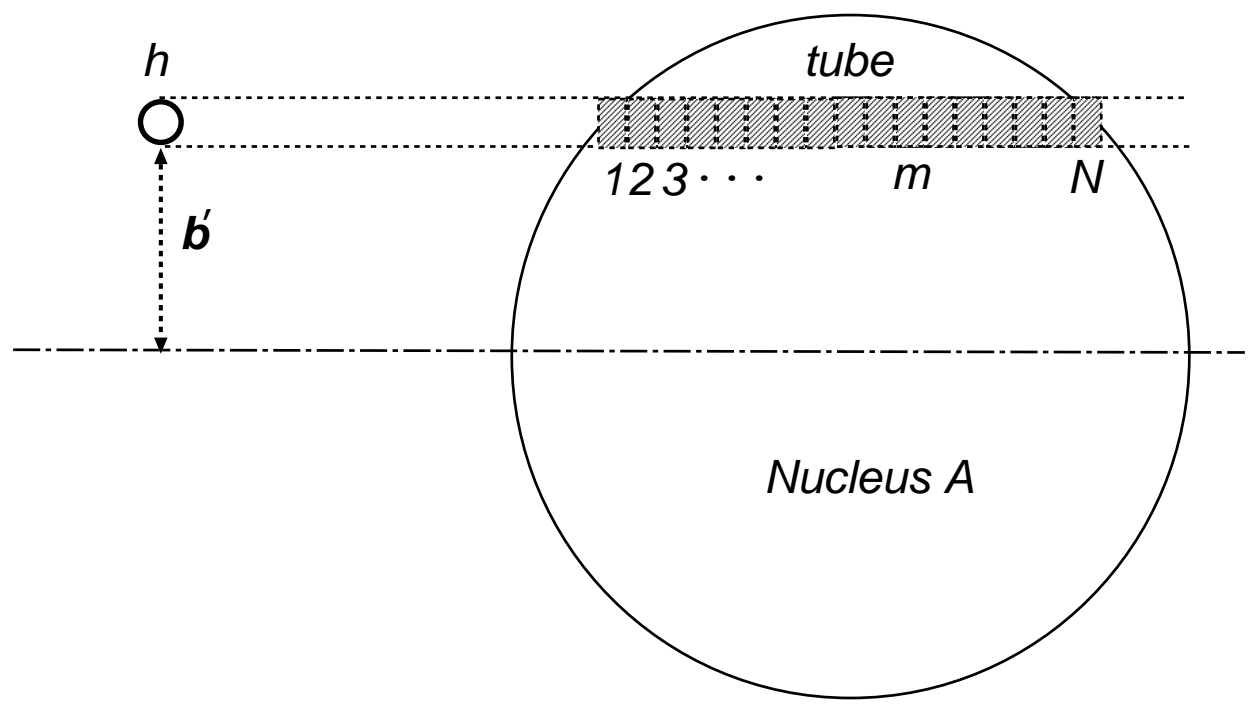

FIG. 1.

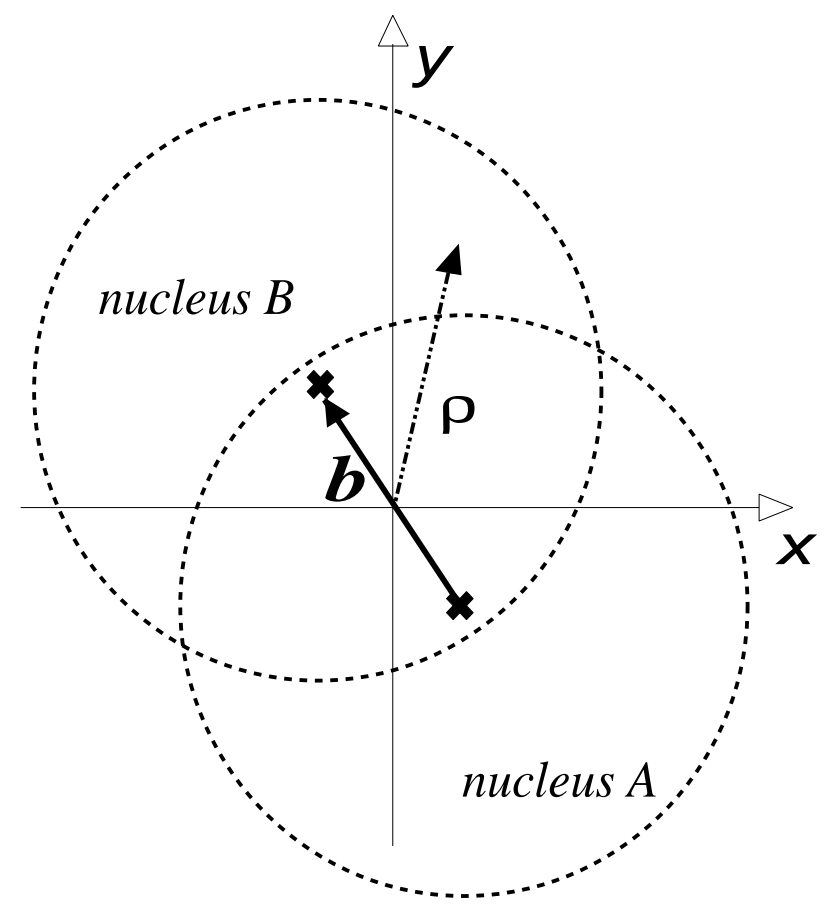

FIG. 2. 


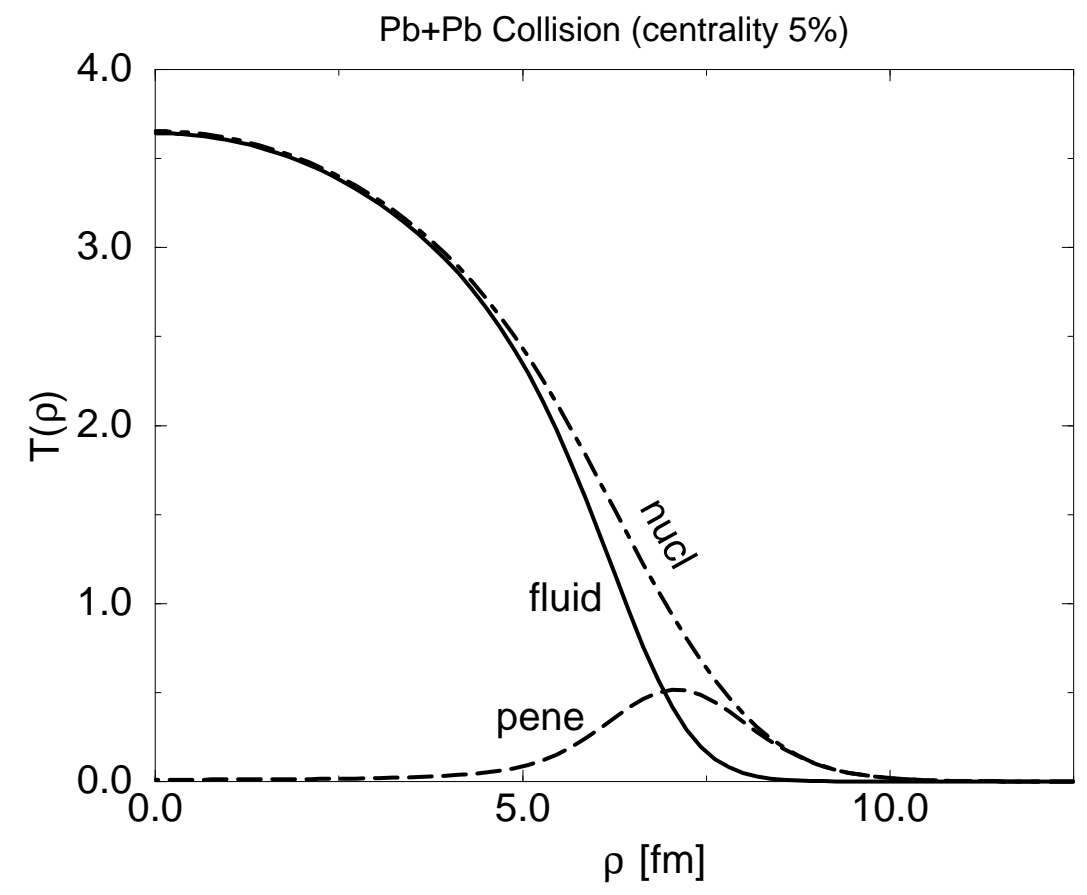

FIG. 3.

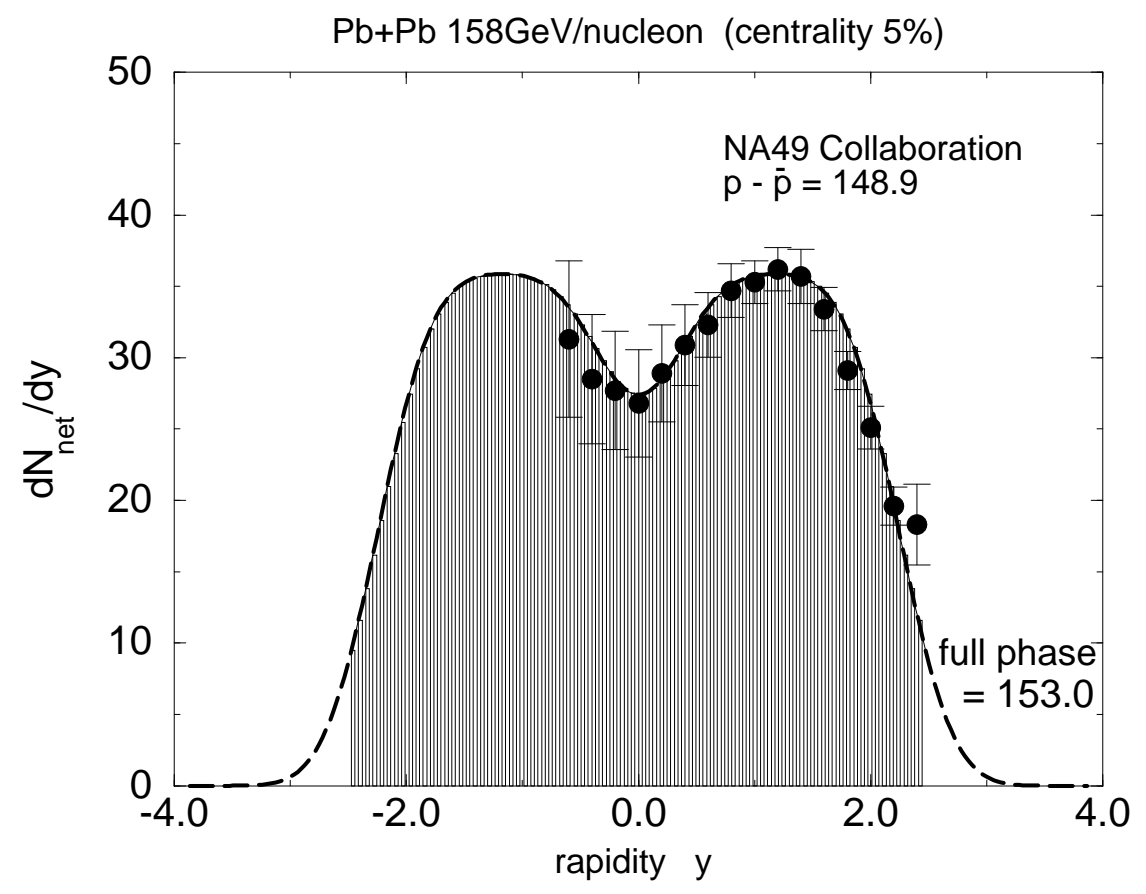

FIG. 4. 


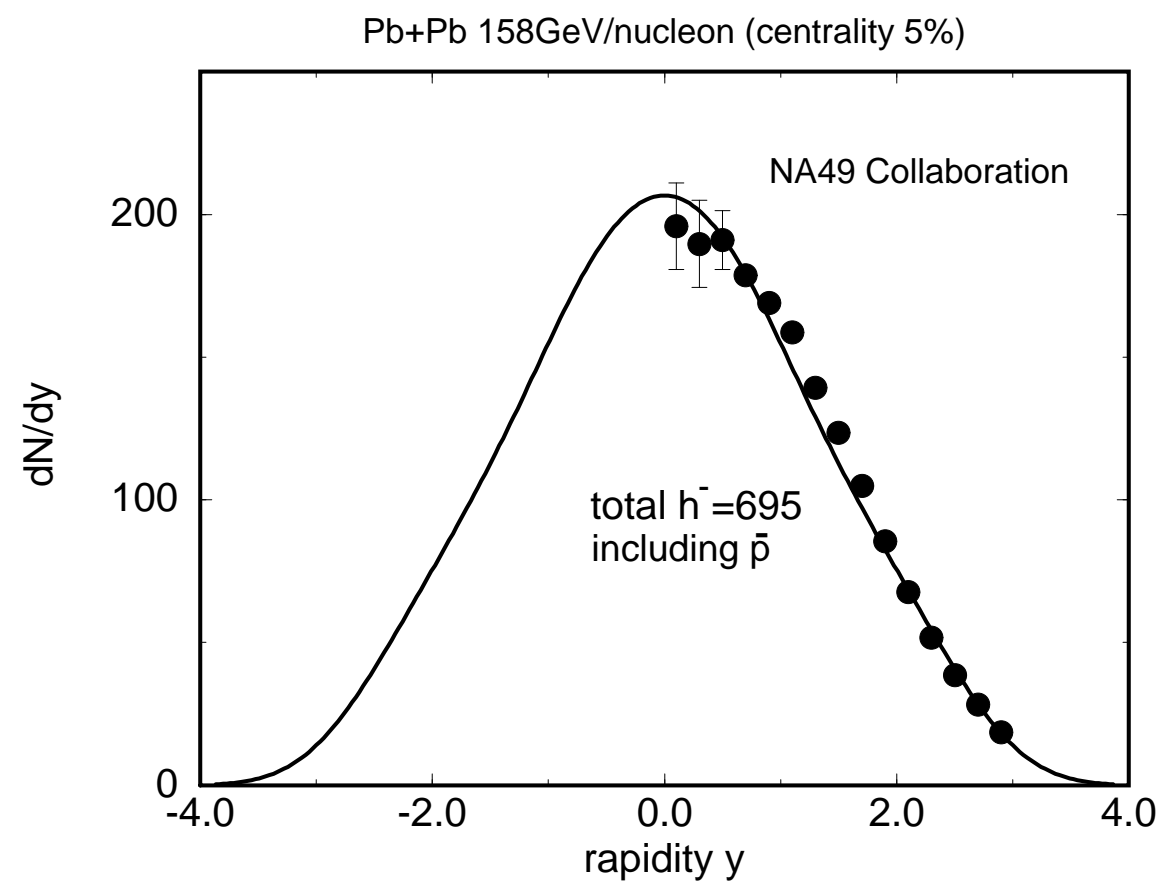

FIG. 5.

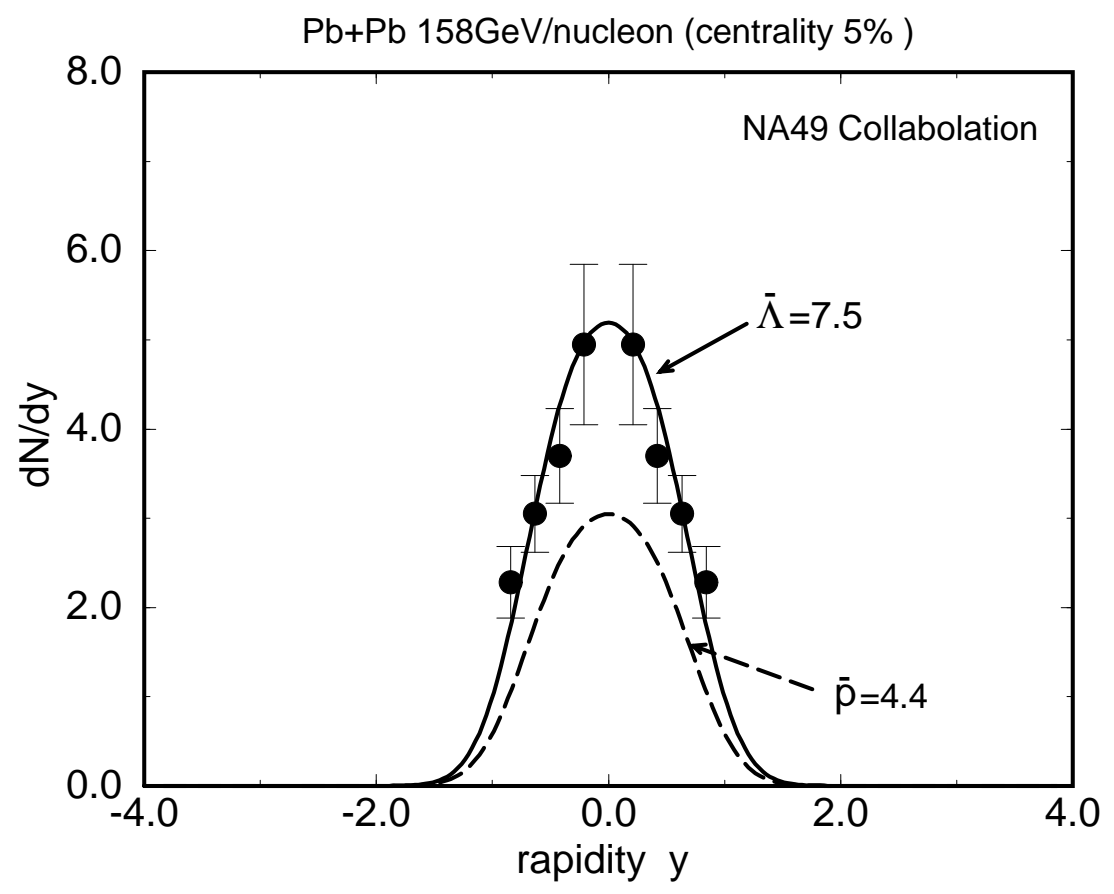

FIG. 6. 


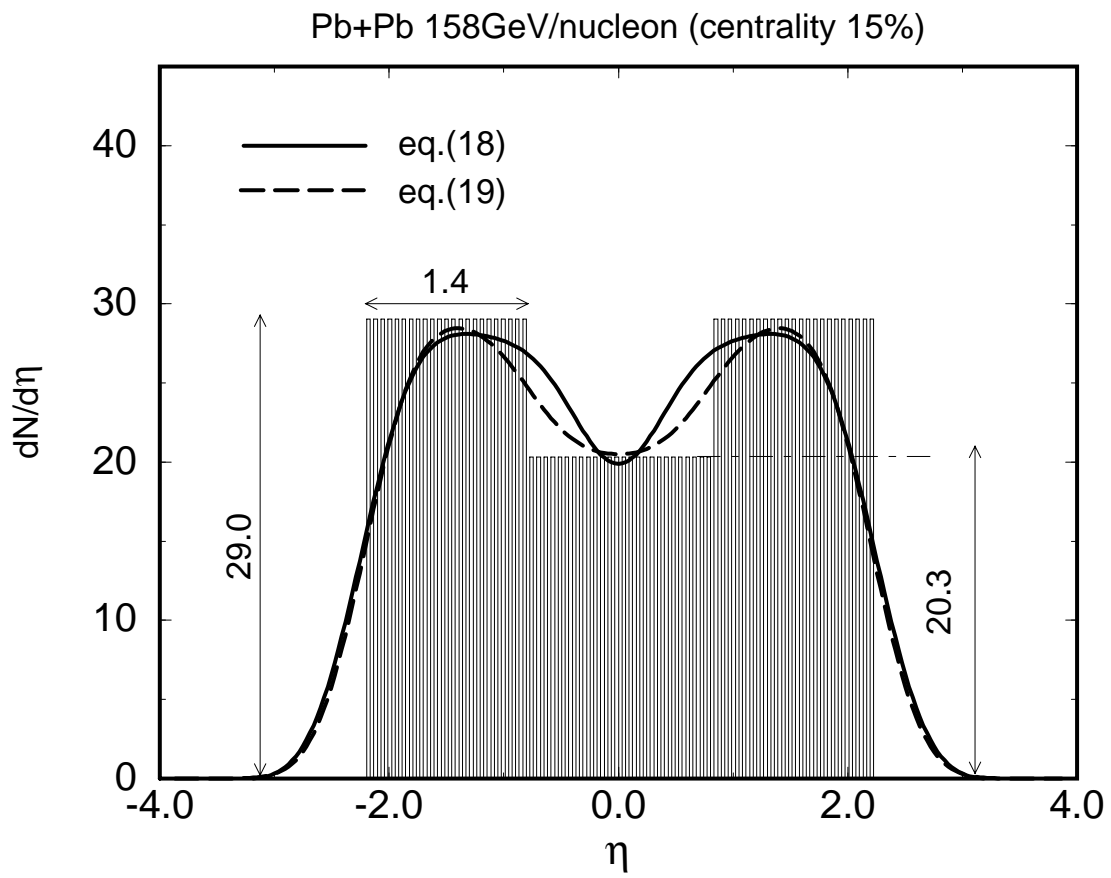

FIG. 7.

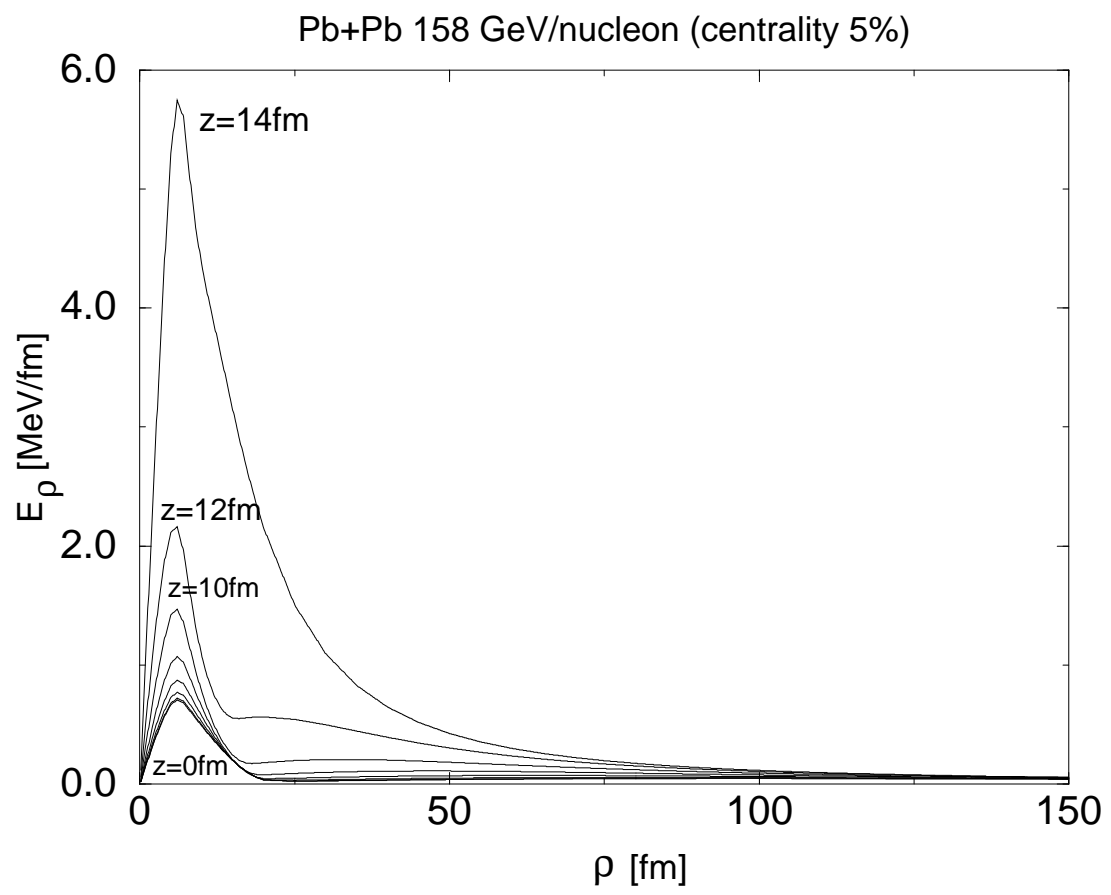

FIG. 8. 


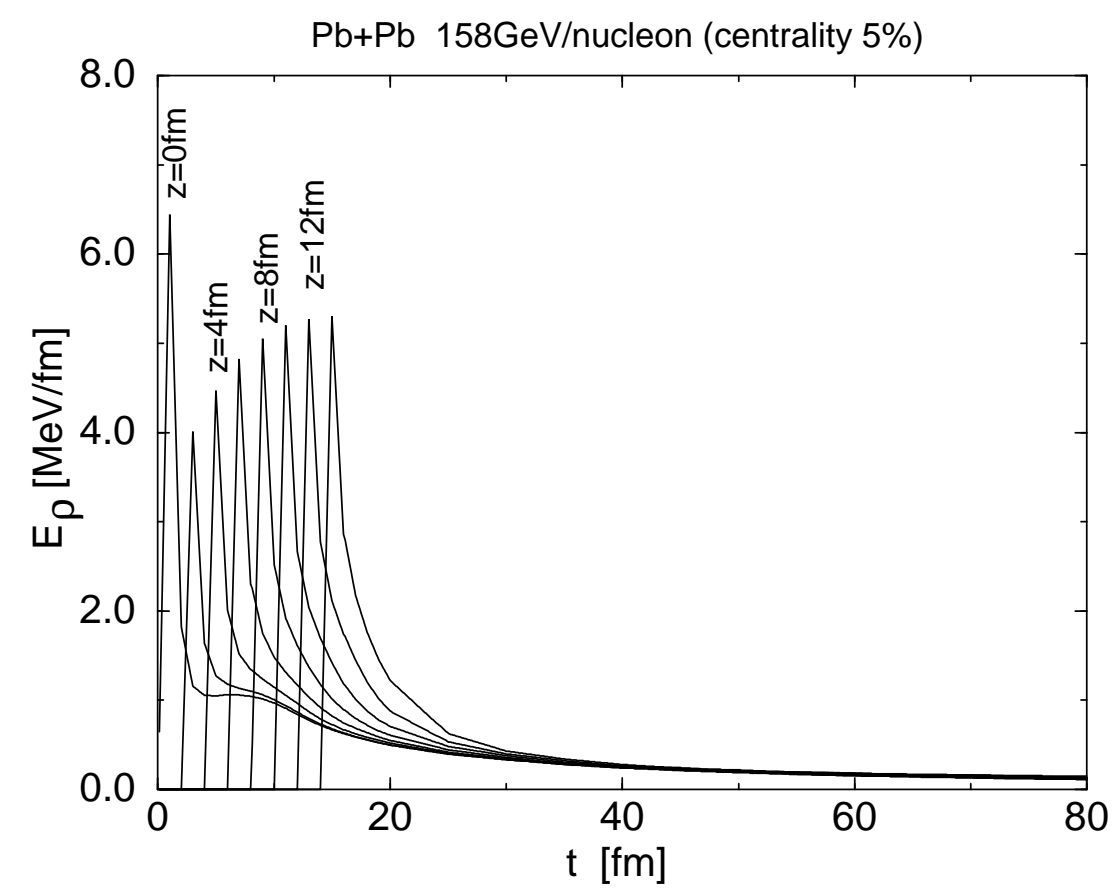

FIG. 9.

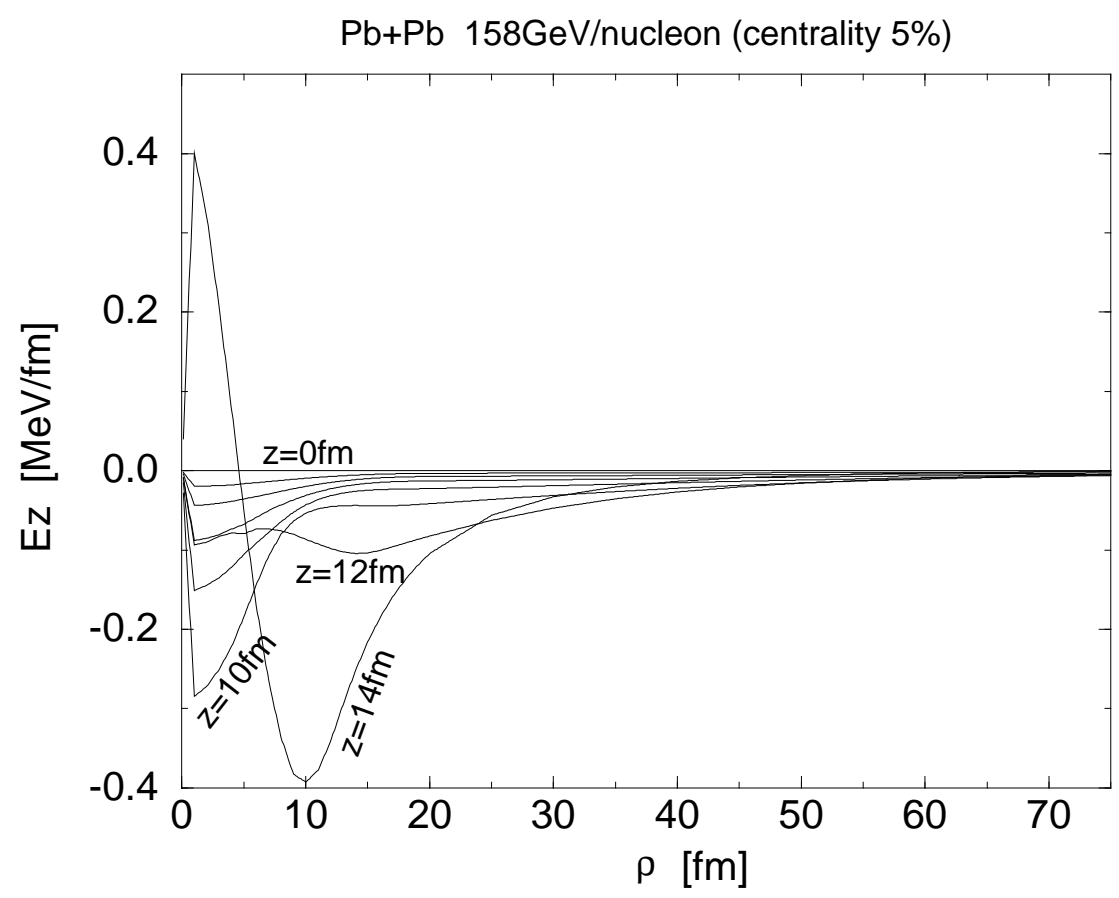

FIG. 10. 


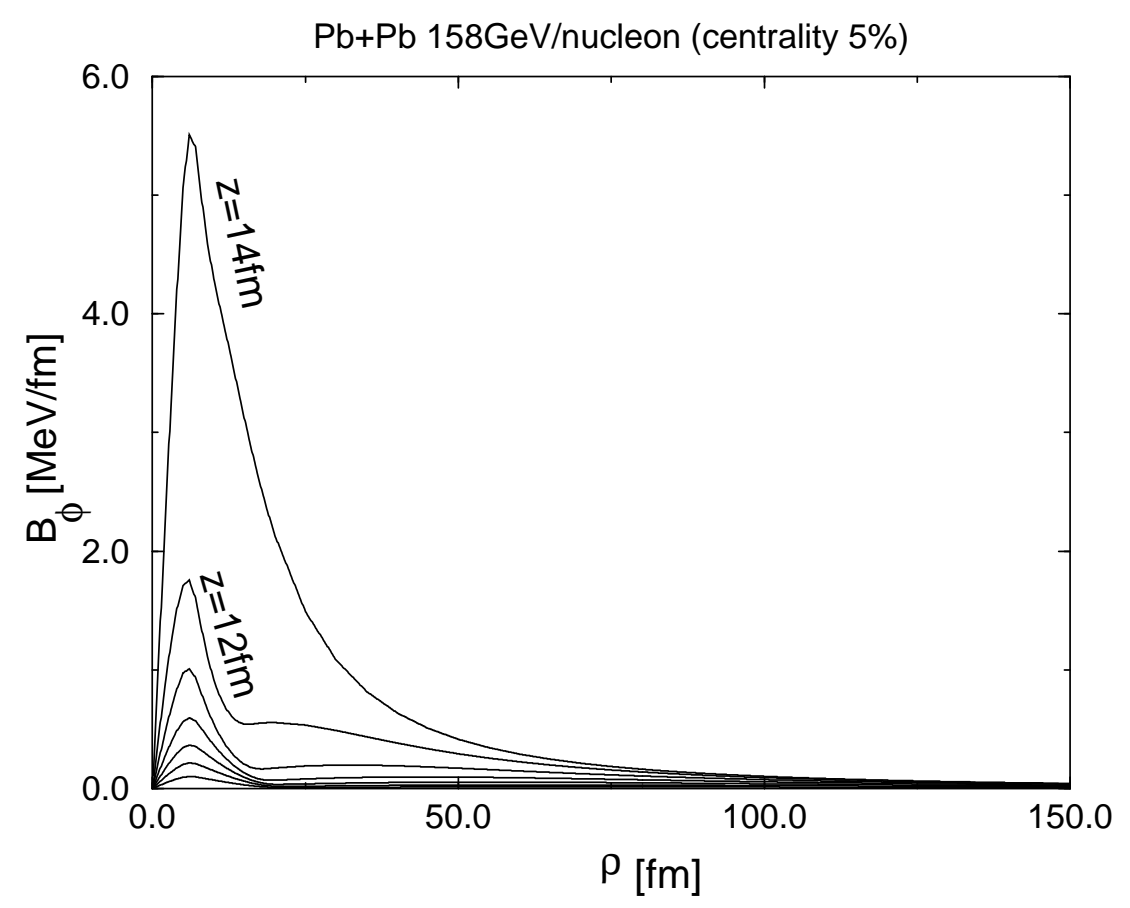

FIG. 11.

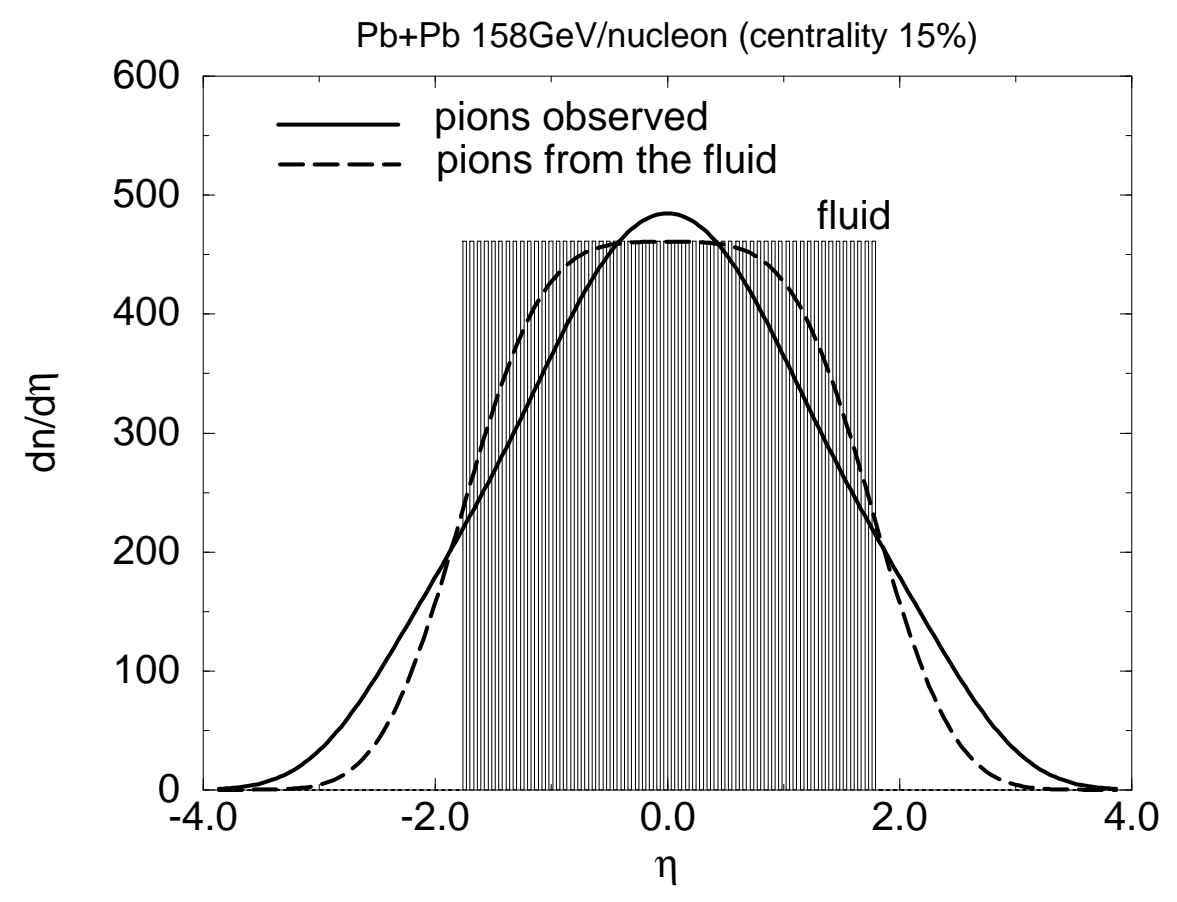

FIG. 12. 


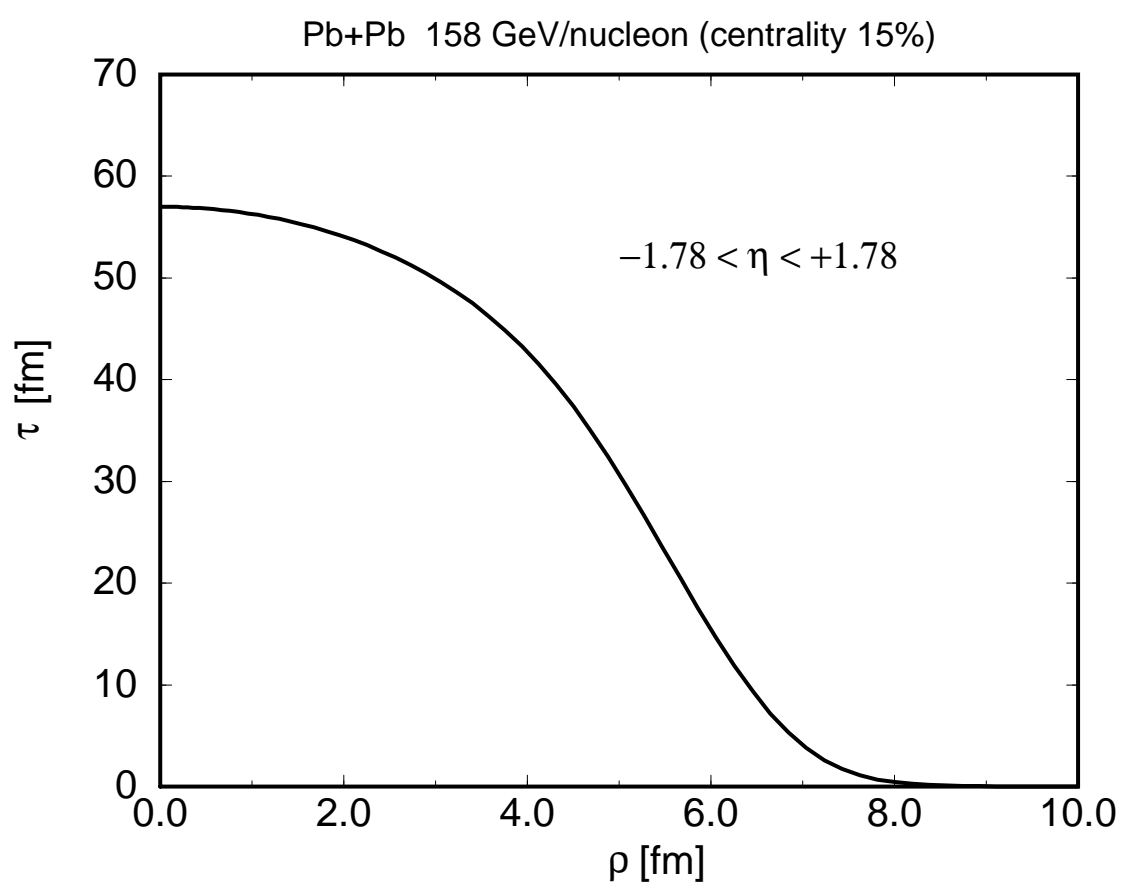

FIG. 13.

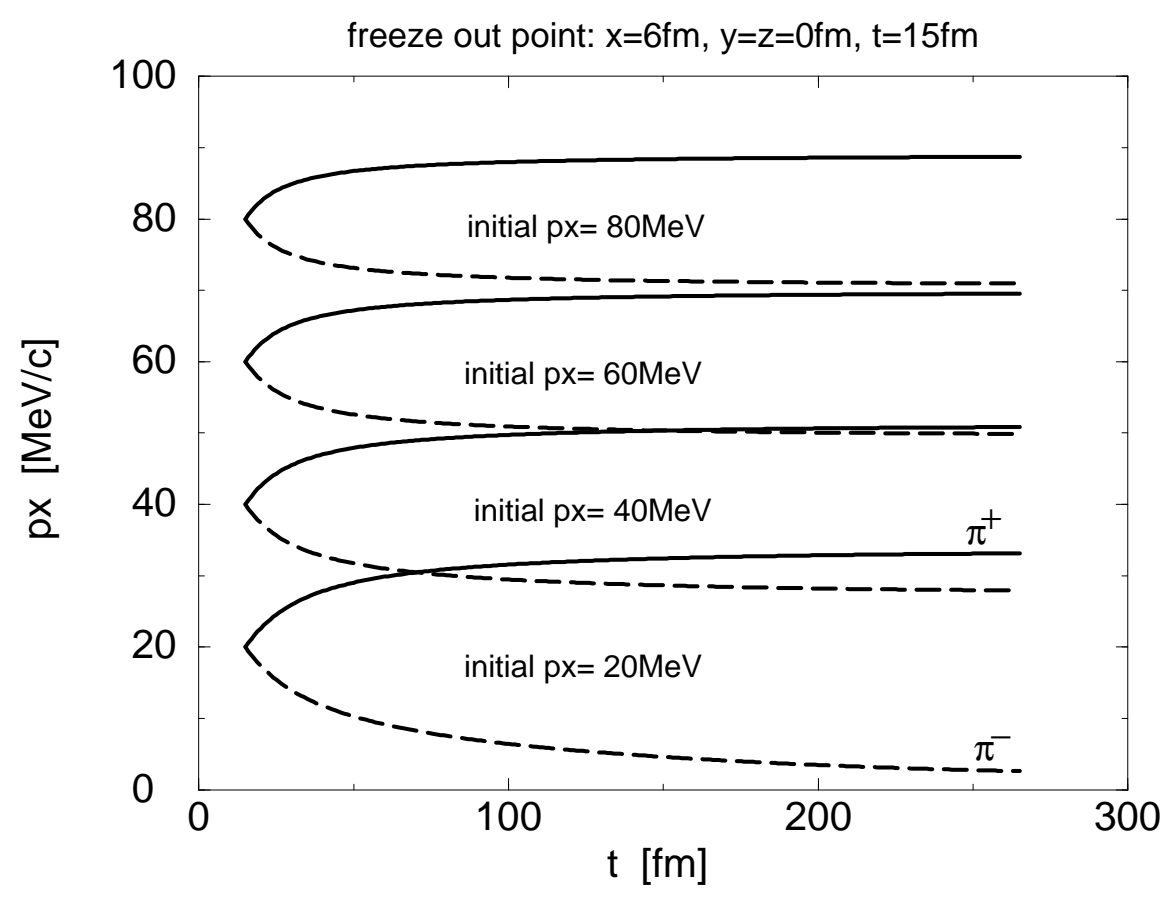

FIG. 14. 


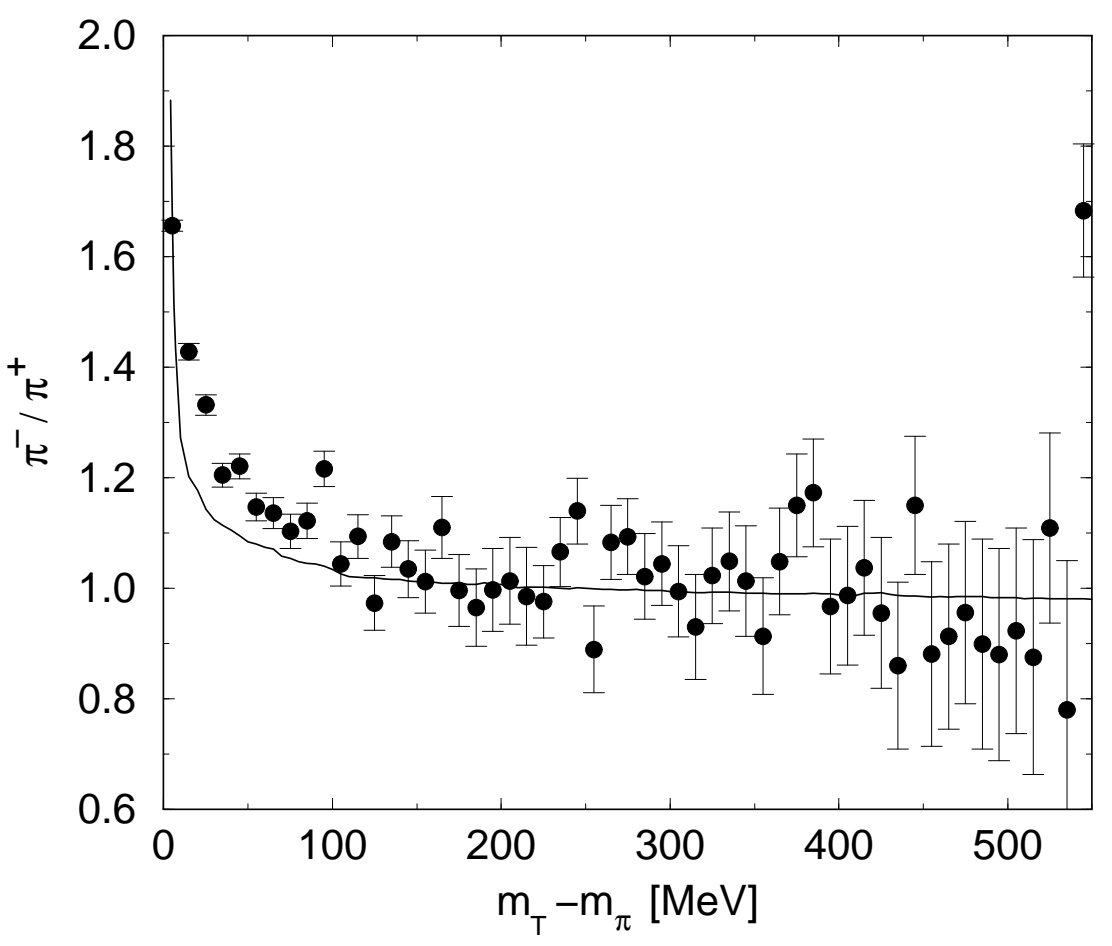

FIG. 15.

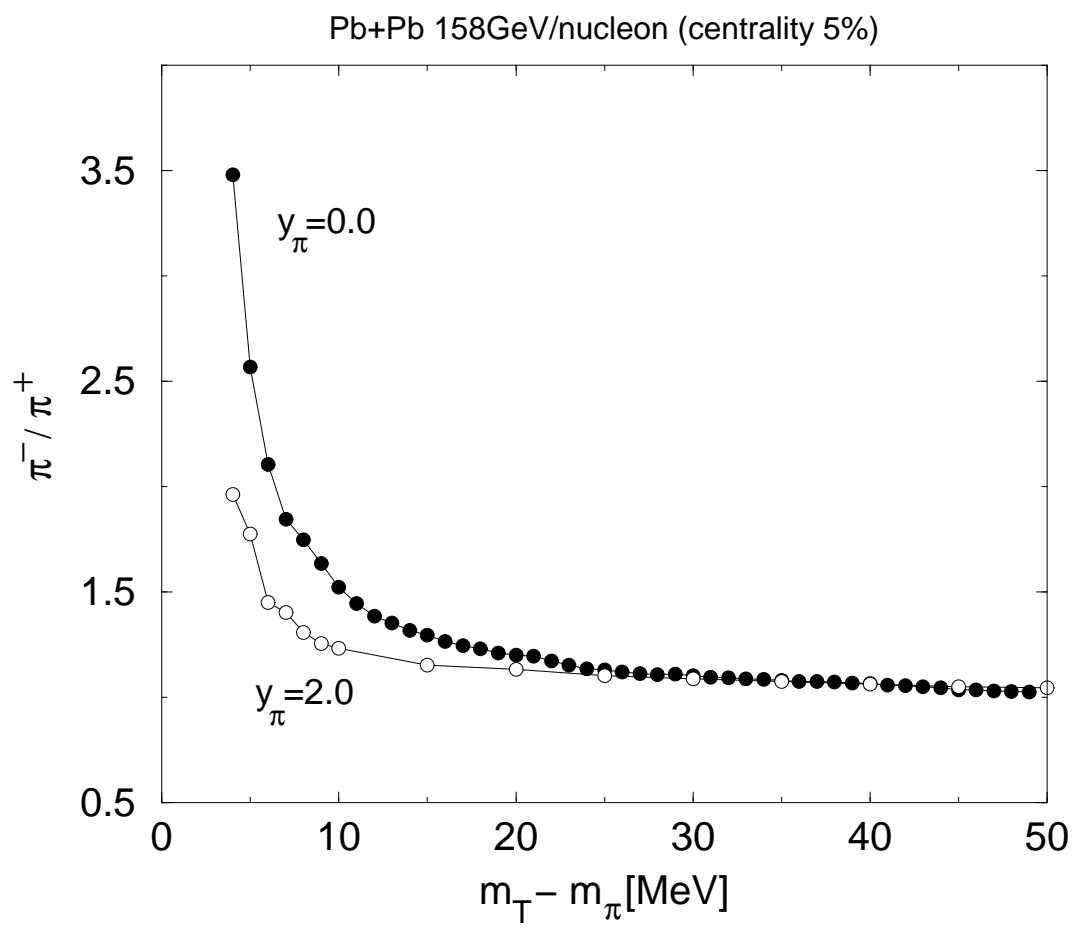

FIG. 16. 


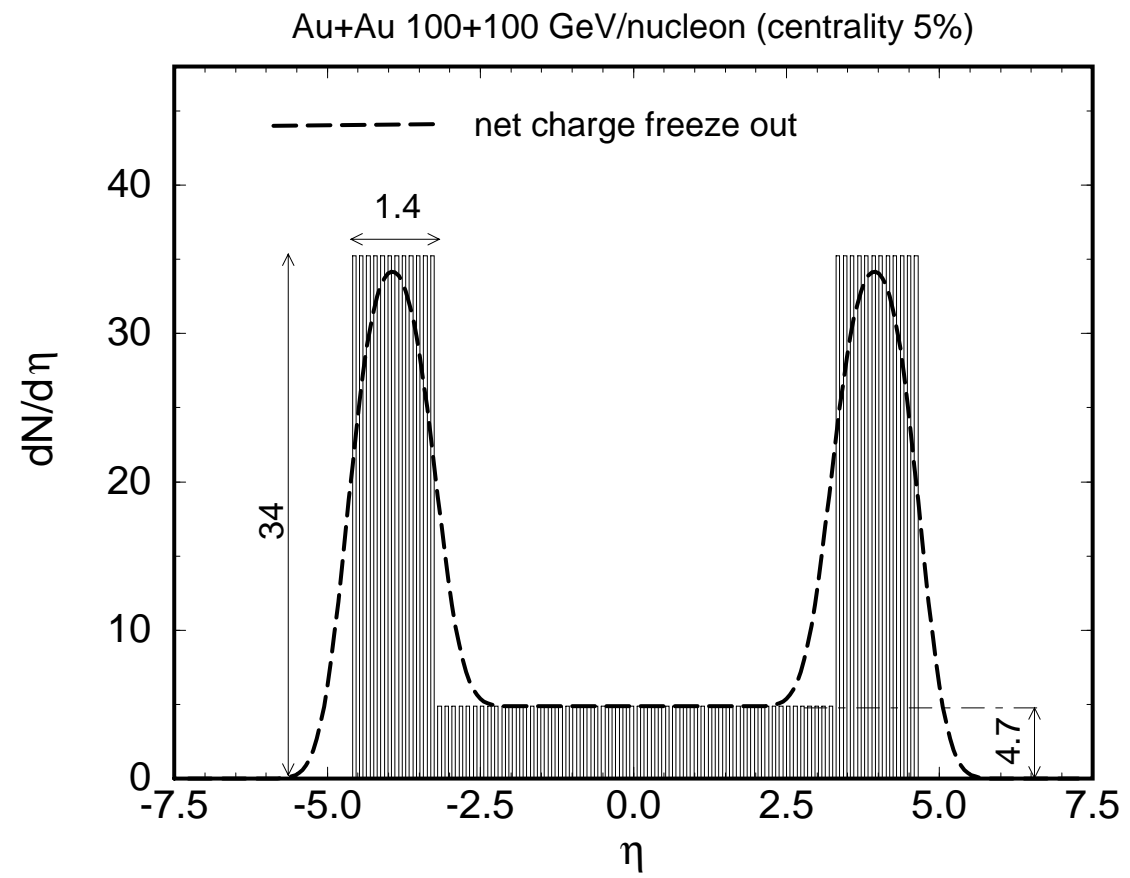

FIG. 17.

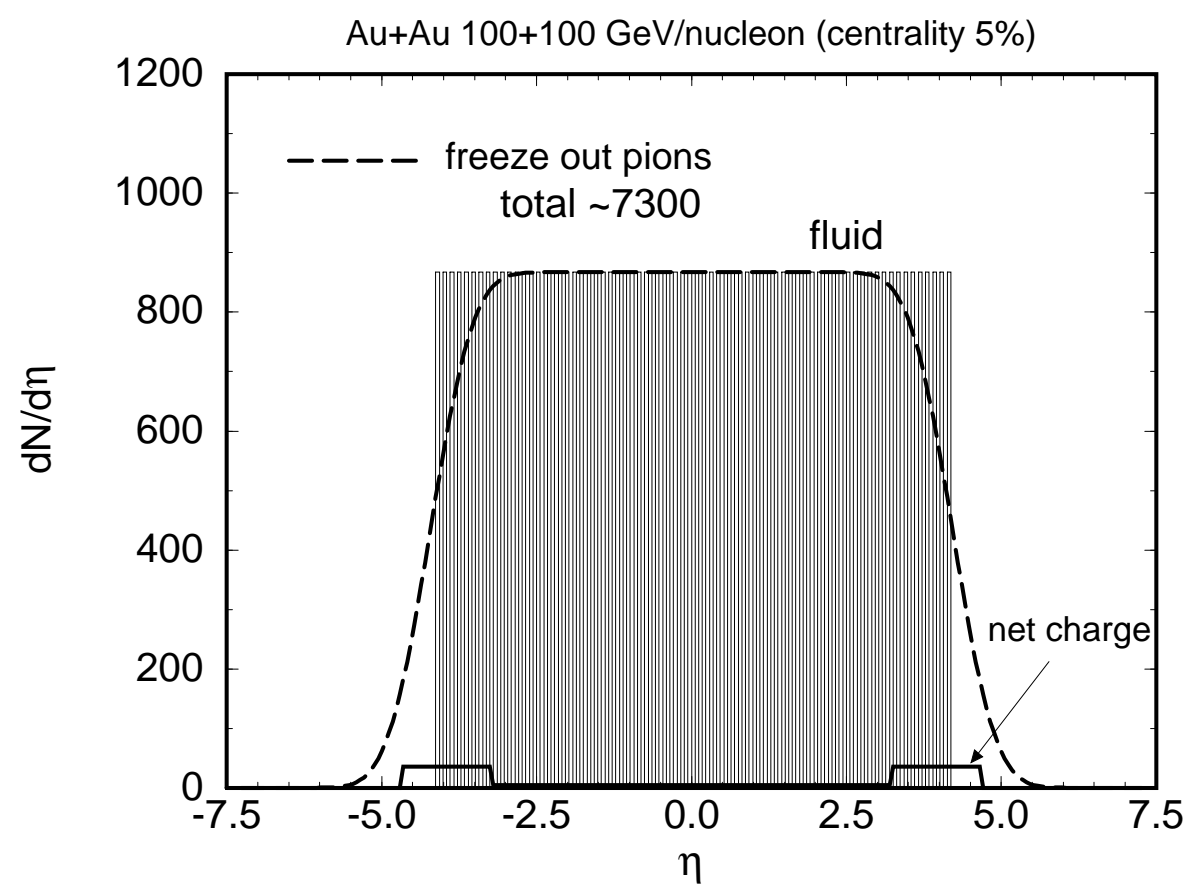

FIG. 18. 


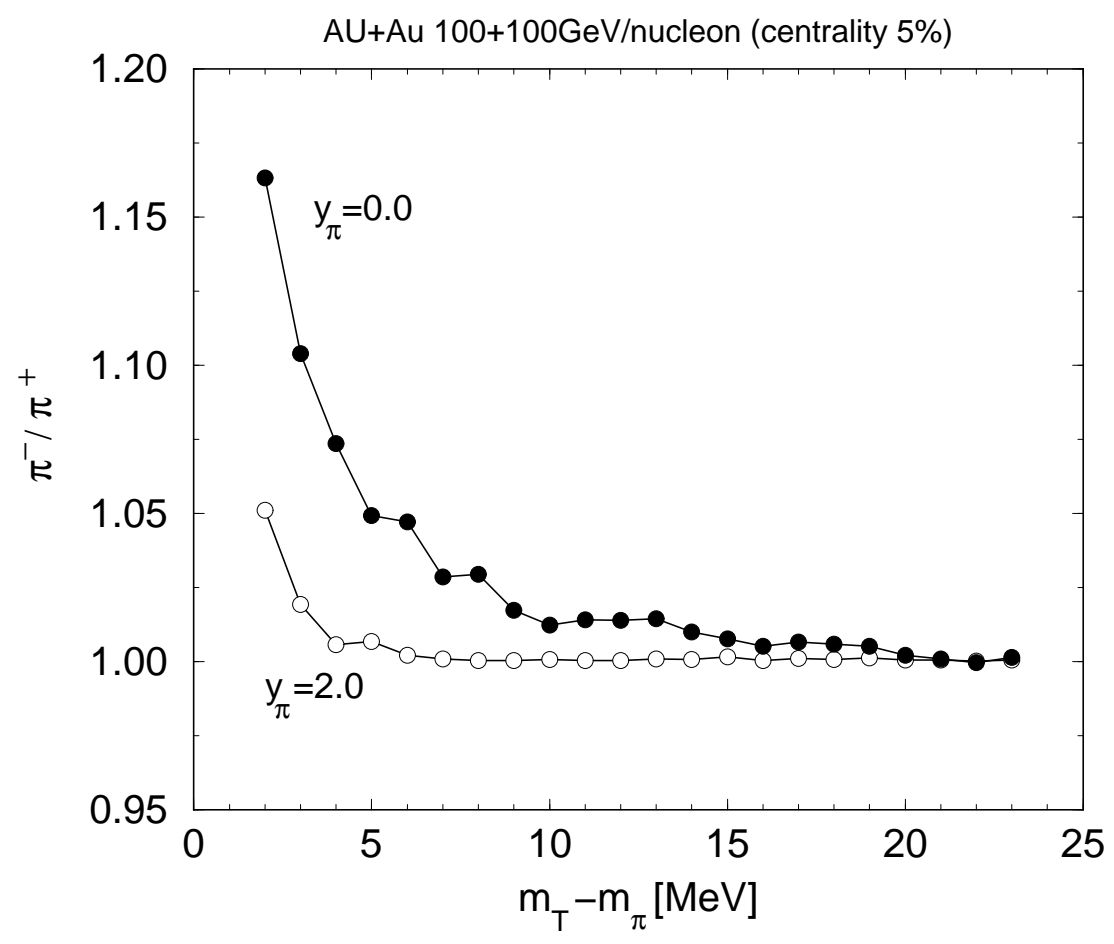

FIG. 19. 